\title{
Is Physiologic Stress Test with Imaging Comparable to Anatomic Examination of Coronary Arteries by Coronary Computed Tomography Angiography to Investigate Coronary Artery Disease? - A Systematic Review and Meta-Analysis
}

Waqas J. Siddiqui ${ }^{1,2}$, Muhammad Shabbir Rawala ${ }^{3}$, Waqas Abid ${ }^{4}$, Muhammad Zain ${ }^{5}$, Murrium I. Sadaf 6, 7 , Danish Abbasi ${ }^{8}$, Chikezie Alvarez ${ }^{9}$, Farah Mansoor ${ }^{10}$, Syed Farhan Hasni ${ }^{11}$, Sandeep Aggarwal 12

1. Cardiology/Nephrology, Drexel University College of Medicine, Philadelphia, USA 2. Cardiology/Nephrology, Orange Park Medical Center, Orange Park, USA 3. Internal Medicine, Charleston Area Medical Center, Charleston, USA 4. Interventional Radiology, Christiana Hospital, Newark, USA 5. Internal Medicine, Sheikh Zayed Medical College and Hospital, Rahim Yar Khan, PAK 6. Internal Medicine, Yale School of Medicine, New Haven, USA 7. Internal Medicine, Waterbury Hospital, Waterbury, USA 8. Cardiovascular Diseases, University of Arkansas, Little Rock, USA 9. Medicine, St. Francis Medical Center, Trenton, USA 10. Medicine, Dow University, Karachi, PAK 11. Heart Failure and Transplant, Albert Einstein Hospital, Philadelphia, USA 12. Nephrology, University of Pennsylvania, Philadelphia, USA

Corresponding author: Waqas J. Siddiqui, dr.waqas20@gmail.com

\section{Abstract \\ Objective}

Coronary computed tomography angiography (CCTA) is a noninvasive diagnostic modality that remains underutilized compared to functional stress testing (ST) for investigating coronary artery disease (CAD). Several patients are misdiagnosed with noncardiac chest pain (CP) that eventually die from a cardiovascular event in subsequent years. We compared CCTA to ST to investigate CP.

\section{Methods}

We searched MEDLINE, PubMed, Cochrane Library, and Embase from January 1, 2007 to July 1, 2018 for randomized controlled trials (RCTs) comparing CCTA to ST in patients who presented with acute or stable CP. We used Review Manager (RevMan) [Computer program] Version 5.3 (Copenhagen: The Nordic Cochrane Centre, The Cochrane Collaboration, 2014) for review and analysis.

\section{Results}

Received 01/24/2020 Review began 02/03/2020 Review ended 02/04/2020 Published 02/10/2020

๑) Copyright 2020

Siddiqui et al. This is an open access article distributed under the terms of the Creative Commons Attribution License CC-BY 4.0., which permits unrestricted use, distribution, and reproduction in any medium, provided the original author and source are credited.
We included 16 RCTs enrolling 21,210 patients; there were more patients with hyperlipidemia and older patients in the ST arm compared to the CCTA arm. There was no difference in mortality: 103 in the CCTA arm vs. 110 in the ST arm (risk ratio $[\mathrm{RR}]=0.93,95 \%$ confidence interval $[\mathrm{CI}]=0.71-1.21, \mathrm{P}=.58$, and $\mathrm{I}^{2}=$ 0\%). A significant reduction was seen in myocardial infarctions (MIs) after CCTA compared to ST: 115 vs. 156 $\left(\mathrm{RR}=0.71, \mathrm{CI}=0.56-0.91, \mathrm{P}<.006, \mathrm{I}^{2}=0 \%\right)$. On subgroup analysis, the CCTA arm had fewer MIs vs. the ST with imaging subgroup $\left(\mathrm{RR}=0.70, \mathrm{CI}=0.54-0.89, \mathrm{P}=.004, \mathrm{I}^{2}=0 \%\right)$ and stable $\mathrm{CP}$ subgroup $(\mathrm{RR}=0.66, \mathrm{CI}=$ $0.50-0.88, \mathrm{P}=.004, \mathrm{I}^{2}=0 \%$ ). The CCTA arm showed significantly higher invasive coronary angiograms and revascularizations and significantly reduced follow-up testing and recurrent hospital visits. A trend towards increased unstable anginas was seen in the CCTA arm.

\section{Conclusions}

Our analysis showed a significant reduction in downstream MIs, hospital visits, and follow-up testing when CCTA is used to investigate CAD with no difference in mortality.

\section{Categories: Cardiology, Radiology}

Keywords: coronary cta, angina, computed tomography angiography, cardiac imaging

\section{Introduction}

Coronary heart disease is one of the leading causes of death, globally. Annually, more than 20 million patients undergo workup for angina [1]. Patients misdiagnosed with noncardiac chest pain (CP) have died from a cardiovascular event five years from the misdiagnosis [2]. Therefore, it is essential to identify patients at the highest risk of coronary artery disease $(\mathrm{CAD})$ who may benefit from a workup using invasive coronary angiography (ICA) and subsequent revascularization. Coronary computed tomography angiography (CCTA) 


\section{Cureus}

is $89 \%$ sensitive and $96 \%$ specific for the diagnosis of CAD, and CCTA is becoming an alternative to ICA due to its comparatively high diagnostic accuracy and noninvasive approach [3-5]. In fact, current cardiology guidelines recommend using CCTA to diagnose CAD [6].

\section{Materials And Methods}

We conducted a systematic review and meta-analysis to compare CCTA to ST with subgroup analyses of ST (with and without imaging which has never been done before) and CP (acute chest pain [ACP] or stable chest pain [SCP]). Over the years, few meta-analyses comparing CCTA to ST have been published, and the outcomes are variable; these are summarized in Table 1 [7-11].

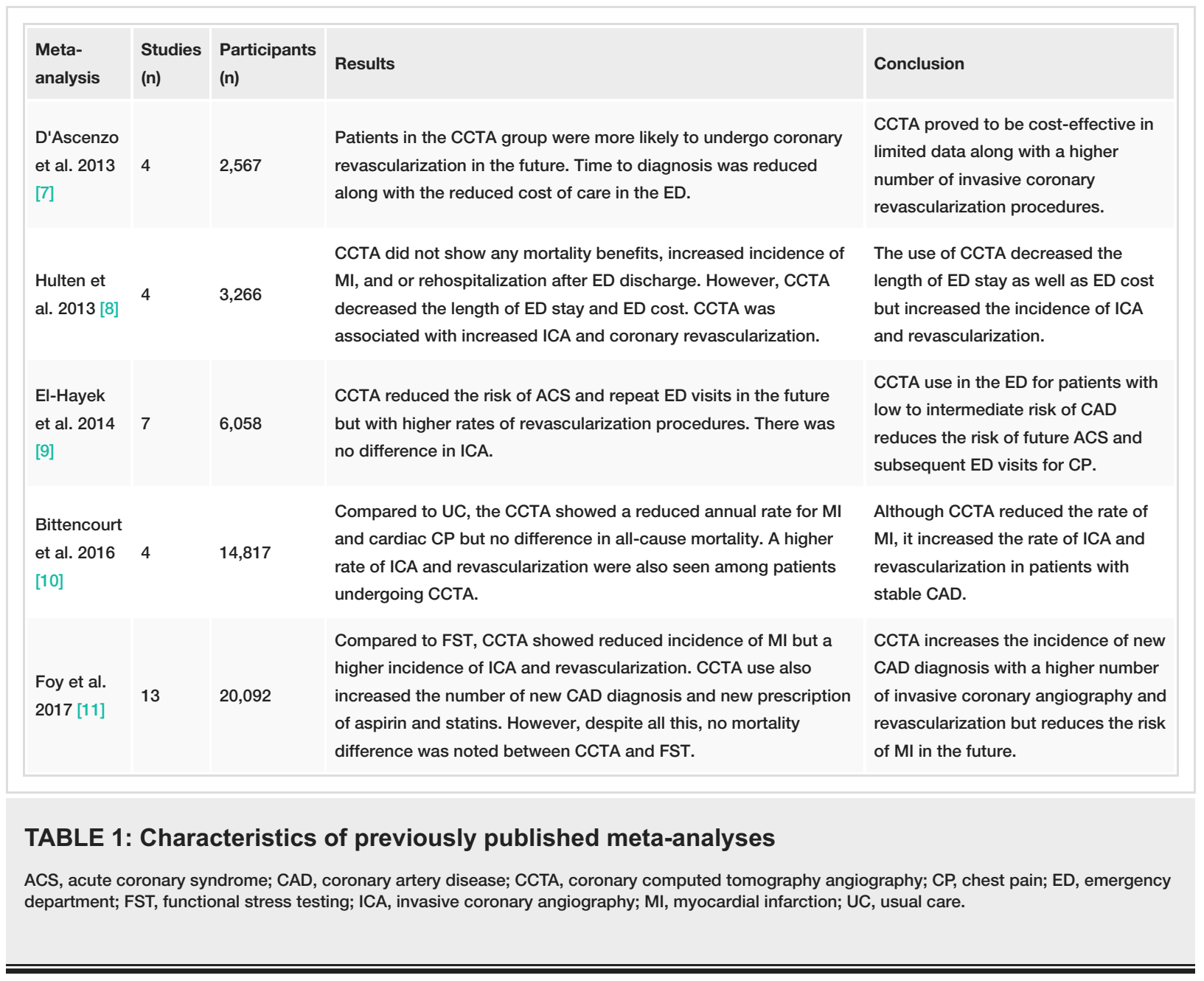

\section{Data sources and searches}

We completed a systematic review according to the Preferred Reporting Items for Systematic Review and Meta-analyses (PRISMA) guidelines [12]. We searched MEDLINE, PubMed, Cochrane Library, and Embase from January 1, 2007 to July 1, 2018 for RCTs, comparing CCTA to ST for suspected underlying CAD in patients who presented with CP. We combined search terms using the Boolean operator OR. Our search strategy included (Coronary Computed Tomography Angiography) OR (CCTA) OR (Coronary CTA) OR (Coronary CT Angiography). Due to the advancement in multislice CT technology, we only included studies performed after 2007. After duplicates were removed, a total of 405 studies were identified.

\section{Study selection}

Three reviewers (W.J.S., W.A., and M.S.R.) reviewed the abstracts and selected 59 articles for a full review. A total of 16 RCTs met the predefined inclusion criteria for qualitative and quantitative analysis comparing CCTA to ST: myocardial perfusion imaging or scan, stress electrocardiogram (bicycle or treadmill), stress echocardiogram, pharmacologic nuclear scan, graded exercise testing, and pharmacologic ST (Figure 1) [1328]. 


\section{Cureus}

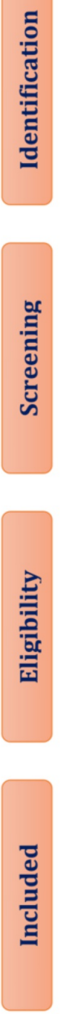

Records identified through
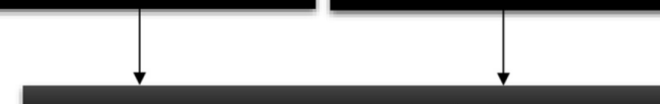

Records after duplicates removed

$(n=405)$
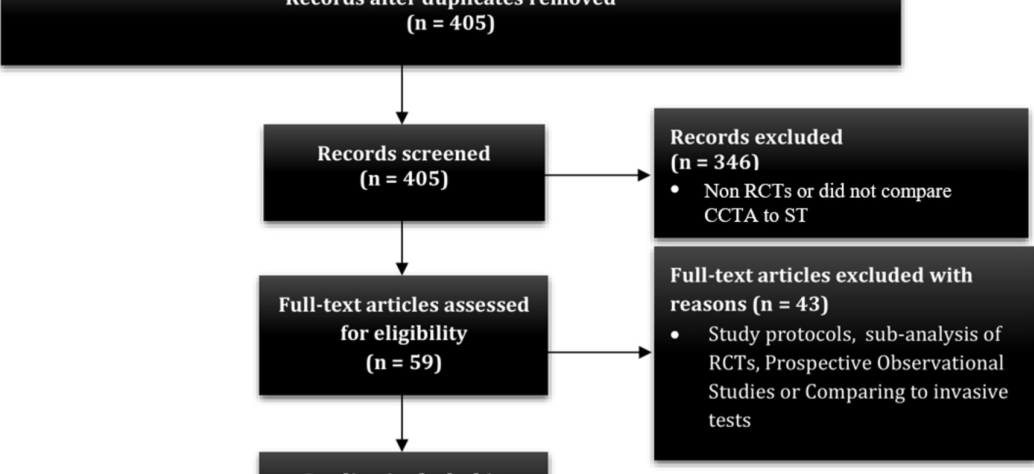

Studies included in

qualitative synthesis ( $n=16)$

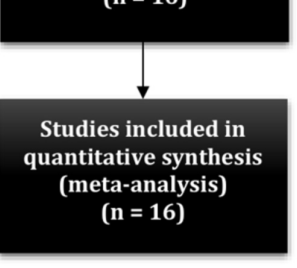

FIGURE 1: PRISMA 2009 study flow diagram

PRISMA, preferred reporting items for systematic reviews and meta-analyses; RCT, randomized control trial; CCTA, coronary computed tomography angiography; ST, stress testing.

\section{Inclusion criteria}

We used the following inclusion criteria: prospective RCTs, RCTs comparing CCTA to ST after CP, age $\geqslant 18$ years, study population $\geqslant 50$ patients, and follow-up $\geqslant$ four weeks.

\section{Data extraction and quality assessment}

W.J.S., M.S.R., and W.A. extracted data into predefined fields on a Microsoft Excel sheet for baseline characteristics and study outcomes. W.J.S. cross-checked the data and made the necessary corrections. All three reviewers discussed the revisions and agreed to the final entry.

\section{Data synthesis and analysis}

\section{Statistical Method}

We used a random-effects model and Mantel-Haenszel method for dichotomous data to calculate the relative risk (RR) and odds ratio (OR), and inverse variance for the continuous data to estimate the standardized mean difference in Review Manager (RevMan) [Computer program] Version 5.3 (Copenhagen: The Nordic Cochrane Centre, The Cochrane Collaboration, 2014). We reported results as forest plots. We used online GraphPad Online Version 8 (GraphPad Software, La Jolla, California, USA) to compare the baseline characteristics and to calculate the number needed to treat (NNT) to prevent one adverse event. A two-tailed P-value of $<.05$ was considered statistically significant. We assumed a 1:1 ratio in each arm except for the ACRIN/PA trial, which randomized patients in a 2:1 ratio where we used the same ratio for outcomes; this failed to uncover any event in both arms [19]. Baseline characteristics are summarized in Table 2 and Table 3 [13-28]. The salient features of each RCT are outlined in Table 4 [13-28]. We used the Cochrane Collaboration's tool for the quality assessment of RCTs (Figure 2, Table 5) [13-28]. 


\begin{tabular}{|c|c|c|c|c|c|c|c|c|c|c|c|}
\hline & Intervention & $\mathbf{n}$ & Age & $\begin{array}{l}\text { Male } \\
\%\end{array}$ & $\begin{array}{l}\text { Female } \\
\%\end{array}$ & $\begin{array}{l}\text { BMI } \\
\left(\mathrm{kg} / \mathrm{m}^{2}\right)\end{array}$ & $\begin{array}{l}\text { HTN } \\
\%\end{array}$ & $\begin{array}{l}\text { HLD } \\
\%\end{array}$ & $\begin{array}{l}\text { DM } \\
\%\end{array}$ & $\begin{array}{l}\text { Smoker } \\
\%\end{array}$ & Aspirin \\
\hline \multirow{2}{*}{ Goldstein et al. 2007 [15] } & ССТА & 99 & $48 \pm 11$ & 43 & 57 & $29 \pm 5$ & 39 & 34 & 8.2 & 15 & 24 \\
\hline & ST & 98 & $51 \pm 12$ & 57 & 43 & $29 \pm 5$ & 38 & 38 & 12.2 & 20 & 29 \\
\hline \multirow{2}{*}{ CT-STAT Goldstein et al. 2011 [16] } & ССТА & 361 & $50 \pm 10$ & 45.2 & 54.8 & $28.1 \pm 4.7$ & 35.5 & 31 & 5.5 & 25.2 & 24.9 \\
\hline & ST & 338 & $50 \pm 10$ & 47 & 53 & $28.7 \pm 5.1$ & 38.8 & 36.1 & 8.3 & 19.5 & 30.5 \\
\hline \multirow{2}{*}{ Miller et-al. 2011 [17] } & CСТА & 30 & $51 \pm 10$ & 43 & 57 & N/A & N/A & N/A & N/A & N/A & N/A \\
\hline & ST & 30 & $51 \pm 10$ & 57 & 43 & N/A & N/A & N/A & N/A & N/A & N/A \\
\hline \multirow{2}{*}{ ACRIN Litt et al. 2012 [19] } & CСТА & 908 & $49 \pm 9$ & 49 & 51 & N/A & 51 & 27 & 14 & 32 & 22 \\
\hline & ST & 462 & $50 \pm 10$ & 44 & 56 & N/A & 50 & 26 & 14 & 34 & 25 \\
\hline \multirow{2}{*}{ Min et al. 2012 [20] } & ССТА & 91 & $55.9 \pm 10$ & 58 & 42 & N/A & 62 & 53 & 23 & 58 & N/A \\
\hline & ST & 89 & $58.9 \pm 9.5$ & 43 & 57 & N/A & 59 & 61 & 21 & 44 & N/A \\
\hline \multirow{2}{*}{ ROMICAT-II Hoffmann et al. 2012 [18] } & CСТА & 501 & $54 \pm 8$ & 52 & 48 & $29.4 \pm 5.3$ & 54 & 46 & 17 & 50 & 23 \\
\hline & ST & 499 & $54 \pm 8$ & 54 & 46 & $29.1 \pm 4.8$ & 54 & 45 & 17 & 49 & 23 \\
\hline \multirow{2}{*}{ CATCH Linde et al. 2013 [21] } & ССТА & 285 & $56.4 \pm 12.2$ & 56.5 & 43.5 & 28 & 47.4 & 41.1 & 12.3 & 60.4 & N/A \\
\hline & ST & 291 & $54.9 \pm 12.2$ & 57.7 & 42.3 & 28 & 36.4 & 34.7 & 10 & 67 & N/A \\
\hline \multirow{2}{*}{$\begin{array}{l}\text { CT-COMPARE Hamilton-Craig et al. } \\
2014 \text { [22] }\end{array}$} & CСТА & 322 & $52.2 \pm 10.7$ & 59 & 41 & N/A & 31 & 25 & 7 & 24 & N/A \\
\hline & ST & 240 & $52.3 \pm 9.8$ & 58 & 42 & N/A & 31 & 24 & 6 & 23 & N/A \\
\hline \multirow{2}{*}{ CAPPA McKavanagh et al. 2015 [26] } & ССТА & 243 & $57.8 \pm 10.0$ & 56.8 & 43.2 & $27.8 \pm 3.6$ & 31.7 & N/A & 5.8 & $19 \%$ & N/A \\
\hline & ST & 245 & $58.9 \pm 10.2$ & 53.5 & 46.5 & $28 \pm 3.6$ & 29.8 & N/A & 4.9 & 19 & N/A \\
\hline \multirow{2}{*}{ PROMISE Douglas et al. 2015 [24] } & CCTA & 4996 & $60.7 \pm 8.3$ & 48.1 & 51.9 & $30.5 \pm 6.1$ & 65 & 67.4 & 21.3 & 50.7 & 45.2 \\
\hline & ST & 5007 & $60.9 \pm 8.3$ & 46.6 & 53.4 & $30.5 \pm 6.1$ & 65 & 67.9 & 21.5 & 51.4 & 44.2 \\
\hline \multirow{2}{*}{ PROSPECT Levsky et al. 2015 [25] } & CCTA & 200 & $56.8 \pm 11.8$ & 37 & 63 & $30.5 \pm 6.2$ & 70.5 & 49 & 33 & 17 & 39 \\
\hline & ST & 200 & $56.3 \pm 10.5$ & 37.5 & 62.5 & $30.7 \pm 6.6$ & 73.5 & 55 & 31 & 13 & 36 \\
\hline \multirow{2}{*}{$\begin{array}{l}\text { SCOT-HEART S-H Investigators } 2015 \\
\text { [23] }\end{array}$} & CCTA & 2073 & $57.1 \pm 9.7$ & N/A & N/A & $29.7 \pm 5.8$ & 34 & 53 & 11 & 53 & 49 \\
\hline & ST & 2073 & $57.0 \pm 9.7$ & N/A & N/A & $29.8 \pm 6$ & 33 & 52 & 11 & 53 & 48 \\
\hline \multirow{2}{*}{ CRESCENT Lubbers et al. 2016 [14] } & CCTA & 242 & $55 \pm 10$ & 45 & 55 & $28 \pm 5$ & 52 & 54 & 17 & 34 & 29 \\
\hline & ST & 108 & $55 \pm 10$ & 44 & 56 & $28 \pm 5$ & 52 & 61 & 16 & 36 & 29 \\
\hline \multirow{2}{*}{ BEACON Dedic et al. 2016 [27] } & CCTA & 250 & $55 \pm 10$ & 51 & 49 & N/A & 36 & 43 & 12 & 47 & 19 \\
\hline & ST & 250 & $53 \pm 9$ & 55 & 45 & N/A & 35 & 45 & 13 & 40 & 14 \\
\hline \multirow{2}{*}{ PERFECT Uretsky et al. 2016 [28] } & CCTA & 206 & $59 \pm 10$ & 46 & 54 & N/A & 68 & 43 & 24 & 45 & 40 \\
\hline & ST & 205 & $60 \pm 10$ & 47 & 53 & N/A & 69 & 53 & 33 & 46 & 44 \\
\hline \multirow{2}{*}{$\begin{array}{l}\text { CRECSCENT-II Lubbers et al. } 2018 \\
\text { [13] }\end{array}$} & CСТА & 130 & $58 \pm 11$ & 51 & 49 & $28 \pm 5$ & 52 & 38 & 18 & 33 & N/A \\
\hline & ST & 138 & $58 \pm 11$ & 44 & 56 & $28 \pm 5$ & 52 & 40 & 18 & 42 & N/A \\
\hline
\end{tabular}

\section{TABLE 2: Baseline characteristics}

BMI, body mass index; CCTA, coronary computed tomography angiography; DM, diabetes mellitus; HLD, hyperlipidemia; HTN, hypertension; N/A, not applicable; ST, stress testing. 


\section{Cureus}

\begin{tabular}{|c|c|c|c|c|c|}
\hline Intervention & ССTA & ST & Mean Difference & $95 \%$ Confidence Interval & P-value \\
\hline $\mathrm{n}$ & 10,937 & 10,273 & & & \\
\hline Age & $57.4 \pm 10$ & $58 \pm 9.8$ & -0.600 & -0.867 to -0.333 & $<.001$ \\
\hline BMI $\left(\mathrm{kg} / \mathrm{m}^{2}\right)$ & $30 \pm 5.9(8,845)$ & $30.1 \pm 5.9(8706)$ & -0.1 & -0.275 to 0.075 & .26 \\
\hline Male \% (n/total) & $49.4(4,379 / 8,864)$ & $49.7(4,075 / 8,200)$ & N/A & N/A & .71 \\
\hline Female \% (n/total) & $50.6(4485 / 8864)$ & $50.3(4,125 / 8,200)$ & N/A & N/A & .71 \\
\hline HTN \% (n/total) & $48.6(5301 / 10,907)$ & $47.8(4,896 / 10,243)$ & $\mathrm{N} / \mathrm{A}$ & $\mathrm{N} / \mathrm{A}$ & .2482 \\
\hline HLD \% (n/total) & $43.2(4,607 / 10,664)$ & $45.6(4,559 / 9,998)$ & N/A & N/A & .0006 \\
\hline DM \% (n/total) & $15.3(1,669 / 10,907)$ & $15.8(1,618 / 10,243)$ & N/A & N/A & .3310 \\
\hline Smoker \% (n/total) & $37.5(4,090 / 10,907)$ & $37.1(3,800 / 10,243)$ & N/A & N/A & .9070 \\
\hline Aspirin \% (n/total) & $31.5(3,127 / 9,927)$ & $32.3(3013 / 9,329)$ & $\mathrm{N} / \mathrm{A}$ & N/A & .2417 \\
\hline
\end{tabular}

\section{TABLE 3: Comparing baseline characteristics}

BMI, body mass index; CCTA, coronary computed tomography angiography; DM, diabetes mellitus; HLD, hyperlipidemia; HTN, hypertension; N/A, not applicable; ST, stress testing.

\begin{tabular}{|c|c|c|c|c|c|c|c|c|c|c|}
\hline Name & Design & Country & $\begin{array}{l}\text { Publication } \\
\text { Year }\end{array}$ & Journal & Enrollment & Population & Setting & $\begin{array}{l}\text { Intervention } \\
\text { vs } \\
\text { Comparison }\end{array}$ & $\begin{array}{l}\text { F/u } \\
\text { Duration }\end{array}$ & CT Scanners \\
\hline $\begin{array}{l}\text { Goldstein et } \\
\text { al. } 2007 \text { [15] }\end{array}$ & RCT & $\begin{array}{l}\text { United } \\
\text { States }\end{array}$ & 2007 & JACC & $\begin{array}{l}\text { March } \\
2005- \\
\text { September } \\
2005\end{array}$ & $\begin{array}{l}\text { Acute } \\
\text { chest pain }\end{array}$ & $\begin{array}{l}\text { Emergency } \\
\text { Department }\end{array}$ & $\begin{array}{l}\text { MSCT vs } \\
\text { rest-stress } \\
\text { MPI }\end{array}$ & $\begin{array}{l}6 \\
\text { months }\end{array}$ & $\begin{array}{l}\text { 64-slice } \\
\text { MSCT } \\
\text { scanner } \\
\text { (Sensation } \\
64 \text { Cardiac, } \\
\text { Siemens } \\
\text { Medical } \\
\text { Systems, } \\
\text { Forchheim, } \\
\text { Germany) }\end{array}$ \\
\hline $\begin{array}{l}\text { CT-STAT } \\
\text { Goldstein et } \\
\text { al. } 2011 \text { [16] }\end{array}$ & $\begin{array}{l}\text { Multicenter, } \\
\text { comparative } \\
\text { effectiveness } \\
\text { RCT }\end{array}$ & $\begin{array}{l}\text { United } \\
\text { States }\end{array}$ & 2011 & JACC & $\begin{array}{l}\text { June } 2007 \\
- \\
\text { November } \\
2008\end{array}$ & $\begin{array}{l}\text { Acute } \\
\text { Chest pain }\end{array}$ & $\begin{array}{l}\text { Emergency } \\
\text { Department }\end{array}$ & $\begin{array}{l}\text { CCTA vs } \\
\text { rest-stress } \\
\text { MPI }\end{array}$ & $\begin{array}{l}6 \\
\text { months }\end{array}$ & $\begin{array}{l}\text { 64-slice } \\
\text { MSCT } \\
\text { scanner } \\
\text { (Sensation } \\
64 \text { Cardiac, } \\
\text { Siemens } \\
\text { Medical } \\
\text { Systems, } \\
\text { Forchheim, } \\
\text { Germany) }\end{array}$ \\
\hline $\begin{array}{l}\text { Miller et al. } \\
2011 \text { [17] }\end{array}$ & $\begin{array}{l}\text { Single- } \\
\text { center RCT }\end{array}$ & $\begin{array}{l}\text { United } \\
\text { States }\end{array}$ & 2011 & $\begin{array}{l}\text { Academic } \\
\text { Emergency } \\
\text { Medicine }\end{array}$ & $\begin{array}{l}\text { October } \\
20,2008- \\
\text { February } \\
02,2009\end{array}$ & $\begin{array}{l}\text { Acute } \\
\text { chest pain }\end{array}$ & $\begin{array}{l}\text { Emergency } \\
\text { Department }\end{array}$ & $\begin{array}{l}\mathrm{SC}+\mathrm{CCTA} \\
\text { vs SC }\end{array}$ & $\begin{array}{l}3 \\
\text { months }\end{array}$ & $\begin{array}{l}\text { 64-slice } \\
\text { multidetector } \\
\text { CT scanner } \\
\text { (Toshiba } \\
\text { America } \\
\text { Medical } \\
\text { Systems, } \\
\text { Inc., Tustin, } \\
\text { CA) }\end{array}$ \\
\hline $\begin{array}{l}\text { ACRIN/PA } \\
\text { Litt et al. } \\
2012 \text { [19] }\end{array}$ & $\begin{array}{l}\text { Multicenter } \\
\text { RCT }\end{array}$ & $\begin{array}{l}\text { United } \\
\text { States }\end{array}$ & 2012 & NEJM & $\begin{array}{l}\text { July 07, } \\
2009 \text { - } \\
\text { November }\end{array}$ & $\begin{array}{l}\text { Acute } \\
\text { Chest pain }\end{array}$ & $\begin{array}{l}\text { Emergency } \\
\text { Department }\end{array}$ & $\begin{array}{l}\text { CCTA vs } \\
\text { Traditional } \\
\text { care }^{\sim}\end{array}$ & 1 month & $\begin{array}{l}\text { 64-slice or } \\
\text { greater } \\
\text { multidetector }\end{array}$ \\
\hline
\end{tabular}




\section{Cureus}

\begin{tabular}{|c|c|c|c|c|c|c|c|c|c|c|}
\hline & & & & & 03, 2011 & & & & & CT scanner \\
\hline $\begin{array}{l}\text { Min et al. } \\
2012 \text { [20] }\end{array}$ & $\begin{array}{l}\text { Multicenter } \\
\text { ( } 2 \text { centers) } \\
\text { RCT }\end{array}$ & $\begin{array}{l}\text { United } \\
\text { States }\end{array}$ & 2012 & JCCT & $\begin{array}{l}\text { December } \\
2008 \text { - } \\
\text { June } 2009\end{array}$ & $\begin{array}{l}\text { Stable } \\
\text { chest pain }\end{array}$ & Outpatient & $\begin{array}{l}\text { CCTA vs. } \\
\text { MPS }\end{array}$ & $\begin{array}{l}2 \\
\text { months }\end{array}$ & $\begin{array}{l}\text { 64-detector } \\
\text { row CT } \\
\text { scanner } \\
\text { (Lightspeed } \\
\text { VCT; GE } \\
\text { Healthcare, } \\
\text { Milwaukee, } \\
\text { WI) }\end{array}$ \\
\hline $\begin{array}{l}\text { ROMICAT-II } \\
\text { Hoffmann et } \\
\text { al. } 2012 \text { [18] }\end{array}$ & $\begin{array}{l}\text { Multicenter } \\
\text { RCT }\end{array}$ & $\begin{array}{l}\text { United } \\
\text { States }\end{array}$ & 2012 & NEJM & $\begin{array}{l}\text { April 23, } \\
2010 \text { - } \\
\text { January } \\
30,2012\end{array}$ & $\begin{array}{l}\text { Acute } \\
\text { chest pain }\end{array}$ & $\begin{array}{l}\text { Emergency } \\
\text { Department }\end{array}$ & $\begin{array}{l}\text { CCTA vs. } \\
\text { SE }\end{array}$ & 28 Days & $\begin{array}{l}\text { 64-slice CT } \\
\text { technology }\end{array}$ \\
\hline $\begin{array}{l}\text { CATCH } \\
\text { Linde et al. } \\
2013 \text { [21] }\end{array}$ & $\begin{array}{l}\text { Single- } \\
\text { center RCT }\end{array}$ & Denmark & 2013 & $\begin{array}{l}\text { International } \\
\text { Journal of } \\
\text { Cardiology }\end{array}$ & $\begin{array}{l}\text { January } \\
2010- \\
\text { January } \\
2013\end{array}$ & $\begin{array}{l}\text { Acute } \\
\text { chest pain }\end{array}$ & $\begin{array}{l}\text { Hospitalized } \\
\text { w/ } \\
\text { suspicion of } \\
\text { NSTE-ACS, } \\
\text { d/c within } \\
24 \text { hours }\end{array}$ & $\begin{array}{l}\text { CCTA vs. } \\
\text { Bicycle } \\
\text { exercise- } \\
\text { ECG and/or } \\
\text { MPI }\end{array}$ & $\begin{array}{l}4 \\
\text { months }\end{array}$ & $\begin{array}{l}320 \\
\text { multidetector } \\
\text { scanner } \\
\text { (Aquilion } \\
\text { One, Toshiba } \\
\text { Medical } \\
\text { systems) }\end{array}$ \\
\hline $\begin{array}{l}\text { CT- } \\
\text { COMPARE } \\
\text { Hamilton- } \\
\text { Craig et al. } \\
2014 \text { [22] }\end{array}$ & $\begin{array}{l}\text { Single- } \\
\text { center RCT }\end{array}$ & Australia & 2014 & $\begin{array}{l}\text { International } \\
\text { Journal of } \\
\text { Cardiology }\end{array}$ & $\begin{array}{l}\text { March } \\
2010 \text {-April } \\
2011\end{array}$ & $\begin{array}{l}\text { Acute } \\
\text { chest pain }\end{array}$ & $\begin{array}{l}\text { Emergency } \\
\text { Department }\end{array}$ & $\begin{array}{l}\text { CCTA vs } \\
\text { Exercise } \\
\text { ECG }\end{array}$ & $\begin{array}{l}12 \\
\text { months }\end{array}$ & $\begin{array}{l}\text { (Somaton } \\
\text { Definition } 64 \\
\text { detector, or } \\
\text { Definition } \\
\text { Flash 128- } \\
\text { detector; } \\
\text { Siemens, } \\
\text { Erlangen, } \\
\text { Germany) }\end{array}$ \\
\hline $\begin{array}{l}\text { CAPPA } \\
\text { McKavanagh } \\
\text { et al. } 2015 \\
\text { [26] }\end{array}$ & $\begin{array}{l}\text { Single- } \\
\text { center RCT }\end{array}$ & Ireland & 2015 & $\begin{array}{l}\text { European } \\
\text { Heart } \\
\text { Journal }\end{array}$ & $\begin{array}{l}\text { September } \\
2010 \text { - } \\
\text { November } \\
2011\end{array}$ & $\begin{array}{l}\text { Stable } \\
\text { chest pain }\end{array}$ & Outpatient & CCT vs. EST & $\begin{array}{l}12 \\
\text { months }\end{array}$ & $\begin{array}{l}\text { 64-detector } \\
\text { platform } \\
\text { (Philips } \\
\text { Brilliance } \\
64 \\
\text { Cleveland, } \\
\text { Ohio, USA) }\end{array}$ \\
\hline $\begin{array}{l}\text { PROMISE } \\
\text { Douglas et } \\
\text { al. } 2015 \text { [24] }\end{array}$ & $\begin{array}{l}\text { Multicenter, } \\
\text { comparative } \\
\text { effectiveness } \\
\text { RCT }\end{array}$ & $\begin{array}{l}\text { United } \\
\text { States }\end{array}$ & 2015 & NEJM & $\begin{array}{l}\text { July } 27, \\
2010 \text { - } \\
\text { September } \\
19,2013\end{array}$ & $\begin{array}{l}\text { Stable } \\
\text { chest pain }\end{array}$ & Outpatient & $\begin{array}{l}\text { CCTA vs. } \\
\text { Functional } \\
\text { testing }\end{array}$ & $\begin{array}{l}25 \\
\text { months }\end{array}$ & $\begin{array}{l}\text { 64-slice or } \\
\text { greater } \\
\text { multidetector } \\
\text { CT scanner }\end{array}$ \\
\hline $\begin{array}{l}\text { PROSPECT } \\
\text { Levsky et al. } \\
2015 \text { [25] }\end{array}$ & $\begin{array}{l}\text { Single- } \\
\text { center, } \\
\text { comparative } \\
\text { effectiveness } \\
\text { RCT }\end{array}$ & $\begin{array}{l}\text { United } \\
\text { States }\end{array}$ & 2015 & $\begin{array}{l}\text { Annals of } \\
\text { Internal } \\
\text { Medicine }\end{array}$ & $\begin{array}{l}\text { July } 2008 \text { - } \\
\text { March } \\
2012\end{array}$ & $\begin{array}{l}\text { Acute } \\
\text { chest pain }\end{array}$ & $\begin{array}{l}\text { Telemetry } \\
\text { Inpatient } \\
\text { Ward }\end{array}$ & $\begin{array}{l}\text { CCTA vs. } \\
\text { MPI }\end{array}$ & $\begin{array}{l}12 \\
\text { months }\end{array}$ & $\begin{array}{l}64 \text {-detector- } \\
\text { row scanners }\end{array}$ \\
\hline $\begin{array}{l}\text { SCOT- } \\
\text { HEART S-H } \\
\text { Investigators } \\
2015 \text { [23] }\end{array}$ & $\begin{array}{l}\text { Open-label, } \\
\text { parallel- } \\
\text { group } \\
\text { Multicenter } \\
\text { RCT }\end{array}$ & Scotland & 2015 & Lancet & $\begin{array}{l}\text { November } \\
18,2010- \\
\text { September } \\
24,2014\end{array}$ & $\begin{array}{l}\text { Stable } \\
\text { chest pain }\end{array}$ & Outpatient & $\begin{array}{l}\text { CCTA + } \\
\text { SOC vs SOC }\end{array}$ & $\begin{array}{l}20 \\
\text { months } \\
(1.7 \\
\text { Years) }\end{array}$ & $\begin{array}{l}\text { 64-row } \\
\text { scanners } \\
\text { (Brilliance 64, } \\
\text { Philips } \\
\text { Medical } \\
\text { Systems, } \\
\text { Biograph } \\
\text { mCT } \\
\text { Siemens) } \\
\text { and 320 } \\
\text { detector row } \\
\text { scanners } \\
\text { (Aquilion } \\
\text { ONE, } \\
\text { Toshiba } \\
\text { Medical }\end{array}$ \\
\hline
\end{tabular}




\section{Cureus}

\begin{tabular}{|c|c|c|c|c|c|c|c|c|c|c|}
\hline & & & & & & & & & & Systems) \\
\hline $\begin{array}{l}\text { CRESCENT } \\
\text { Lubbers et } \\
\text { al. } 2016 \text { [14] }\end{array}$ & $\begin{array}{l}\text { Multicenter } \\
\text { RCT }\end{array}$ & Netherland & 2016 & $\begin{array}{l}\text { European } \\
\text { Heart } \\
\text { Journal }\end{array}$ & $\begin{array}{l}\text { April } 2011 \\
\text { - July } 2013\end{array}$ & $\begin{array}{l}\text { Stable } \\
\text { chest pain }\end{array}$ & Outpatient & $\begin{array}{l}\text { CCT vs. } \\
\text { Functional } \\
\text { testing }\end{array}$ & $\begin{array}{l}12 \\
\text { months }\end{array}$ & $\begin{array}{l}64 \text {-slice or } \\
\text { more } \\
\text { advanced CT } \\
\text { technology, } \\
\text { with } \\
\text { radiation } \\
\text { minimizing } \\
\text { measures }\end{array}$ \\
\hline $\begin{array}{l}\text { BEACON } \\
\text { Dedic et al. } \\
2016 \text { [27] }\end{array}$ & $\begin{array}{l}\text { Multicenter, } \\
\text { Prospective, } \\
\text { open-label, } \\
\text { RCT }\end{array}$ & Netherland & 2016 & JACC & $\begin{array}{l}\text { July 11, } \\
2011 \text { - } \\
\text { January } \\
30,2014\end{array}$ & $\begin{array}{l}\text { Acute } \\
\text { chest pain }\end{array}$ & $\begin{array}{l}\text { Emergency } \\
\text { Department }\end{array}$ & $\begin{array}{l}\text { CCTA vs. } \\
\text { soc }^{\Psi}\end{array}$ & 30 days & $\begin{array}{l}\text { 64-slice or } \\
\text { more } \\
\text { advanced CT } \\
\text { technology, } \\
\text { using ECG- } \\
\text { synchronized } \\
\text { axial or spiral } \\
\text { scan } \\
\text { protocols }\end{array}$ \\
\hline $\begin{array}{l}\text { PERFECT } \\
\text { Uretsky et al. } \\
2016 \text { [28] }\end{array}$ & $\begin{array}{l}\text { Single- } \\
\text { center, } \\
\text { comparative } \\
\text { effectiveness } \\
\text { RCT }\end{array}$ & $\begin{array}{l}\text { United } \\
\text { States }\end{array}$ & 2016 & $\begin{array}{l}\text { Journal of } \\
\text { Nuclear } \\
\text { Cardiology }\end{array}$ & $\begin{array}{l}\text { July } 2011 \text { - } \\
\text { December } \\
2013\end{array}$ & $\begin{array}{l}\text { Acute } \\
\text { chest pain }\end{array}$ & Inpatient & $\begin{array}{l}\text { CCTA vs. } \\
\text { Stress Test }\end{array}$ & $\begin{array}{l}12 \\
\text { months }\end{array}$ & $\begin{array}{l}\text { (Toshiba } \\
\text { Aquilion 64- } \\
\text { detector } \\
\text { Toshiba } \\
\text { America } \\
\text { Medical } \\
\text { Systems, } \\
\text { Tustin, CA, or } \\
\text { Siemens } \\
\text { Somatoform } \\
\text { Sensation } \\
\text { 64-detector, } \\
\text { Siemens } \\
\text { Medical } \\
\text { Solutions } \\
\text { USA, } \\
\text { Malvern, PA). }\end{array}$ \\
\hline $\begin{array}{l}\text { CRESCENT- } \\
\text { II Lubbers et } \\
\text { al. } 2017 \text { [13] }\end{array}$ & $\begin{array}{l}\text { Multicenter } \\
\text { RCT }\end{array}$ & Netherland & 2017 & JACC & $\begin{array}{l}\text { July } 2013 \text { - } \\
\text { November } \\
2015\end{array}$ & $\begin{array}{l}\text { Stable } \\
\text { Angina }\end{array}$ & Outpatient & $\begin{array}{l}\text { CCT vs. } \\
\text { Functional } \\
\text { testing }\end{array}$ & $\begin{array}{l}6 \\
\text { months }\end{array}$ & $\begin{array}{l}\text { Somatom } \\
\text { Definition } \\
\text { Flash and } \\
\text { Force } \\
\text { Siemens } \\
\text { Healthineers, } \\
\text { Forchheim, } \\
\text { Germany }\end{array}$ \\
\hline
\end{tabular}

\section{TABLE 4: Characteristics of randomized control trials}

$\sim$ Traditional Care $=$ Graded exercise testing/Pharmacologic stress testing

* Stress Test $=$ Stress Echocardiography/MPI

\# Functional testing $=$ Exercise ECG, Exercise or Pharmacologic Nuclear Stress Testing, and Stress Echocardiography

$\square \mathrm{SOC}=$ Standard Optimal Care

CCT, cardiac computerized tomography; CCTA, coronary computed tomography angiography; ECG, electrocardiography; EST, exercise stress electrocardiography test; F/u, follow up; JACC, Journal of American College of Cardiology; JCCT, Journal of Cardiovascular Computed Tomography; MPI, myocardial perfusion imaging; MPS, myocardial perfusion scan; MSCT, multi-slice computed tomographic angiography; NEJM, New England Journal of Medicine; NSTE-ACS, non-ST elevated acute coronary syndrome; RCT, randomized control trial; SC, standard care; SE, standard evaluation; SOC, standard of care; w/, with. 


\section{Cureus}

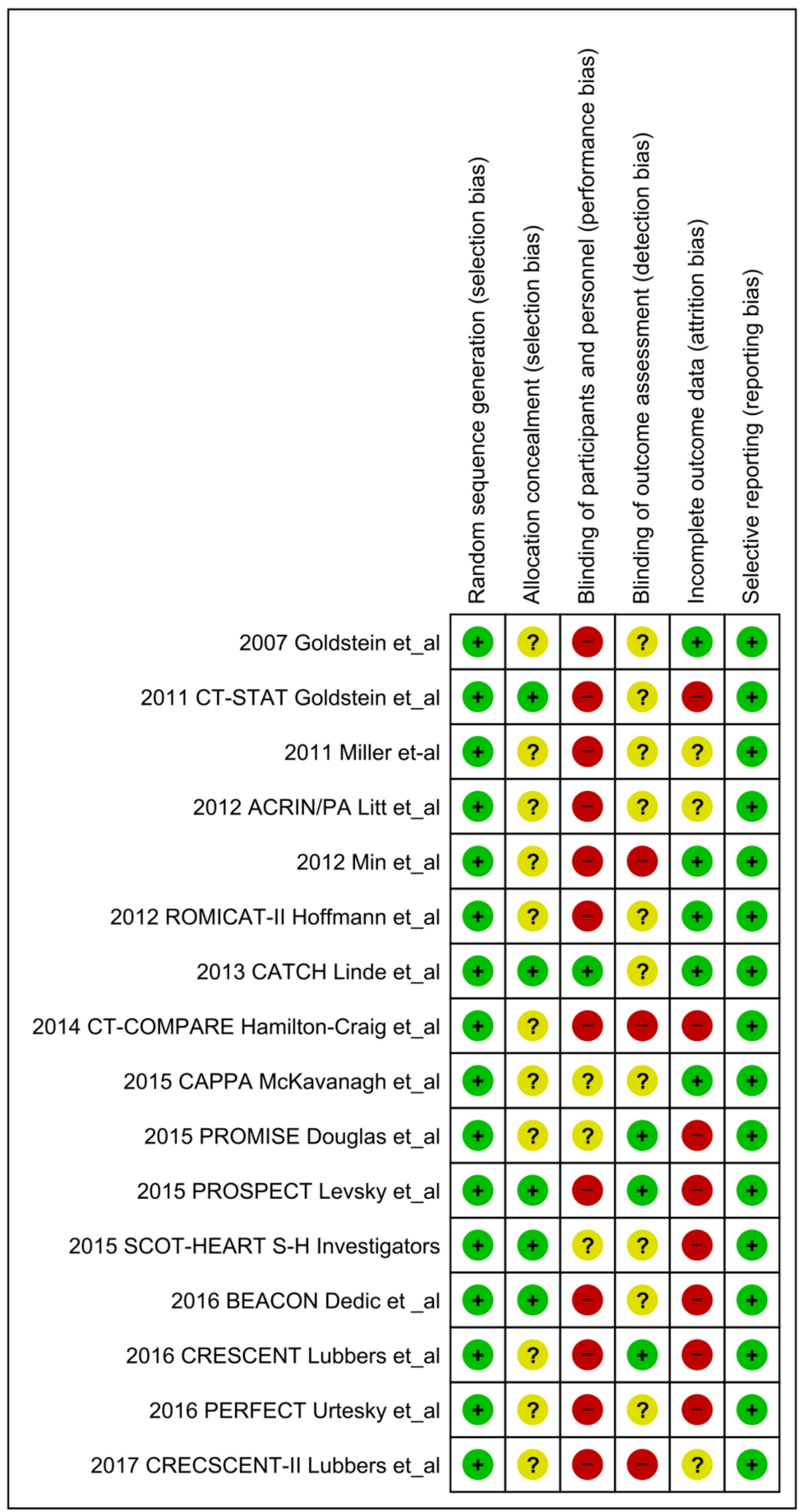

FIGURE 2: Cochrane Collaboration's tool for the quality assessment of randomized controlled trials

Bias risk presented in 16 studies [13-28]

\begin{tabular}{|l|l|l|l|l|l|}
\hline Name & Random Sequence & $\begin{array}{l}\text { Allocation } \\
\text { Concealment }\end{array}$ & $\begin{array}{l}\text { Blinding of } \\
\text { Participants and } \\
\text { Personnel }\end{array}$ & $\begin{array}{l}\text { Blinding of } \\
\text { Outcome } \\
\text { Assessment }\end{array}$ & $\begin{array}{l}\text { Incomplete } \\
\text { Outcome } \\
\text { Data }\end{array}$ \\
\hline $\begin{array}{l}\text { Goldstein et al. } 2007 \\
{[15]}\end{array}$ & $\begin{array}{l}\text { Yes via SAS software } \\
\text { version } 9.1\end{array}$ & Not reported & No & Not reported \\
Bias
\end{tabular}




\section{Cureus}

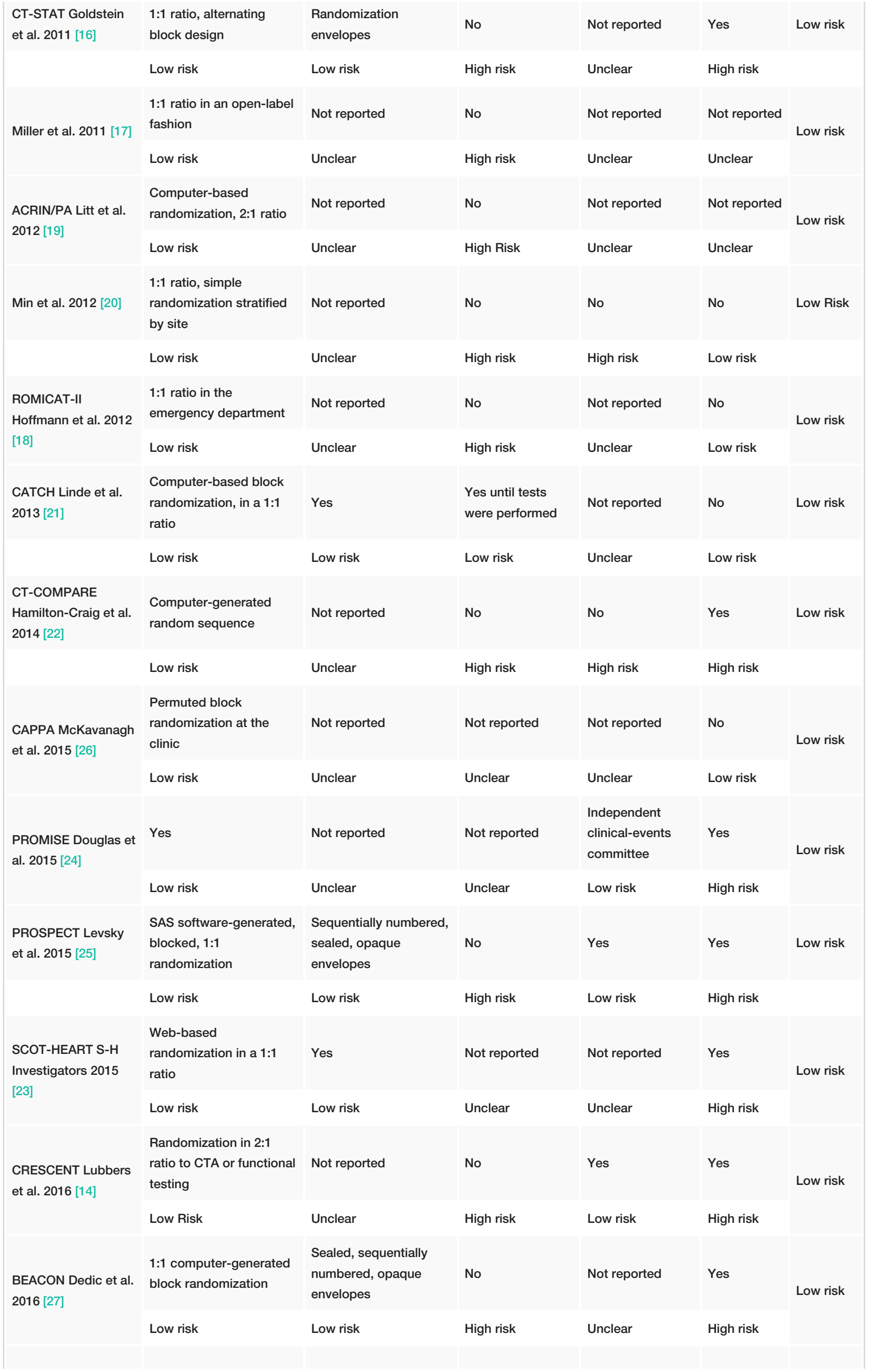




\section{Cureus}

\begin{tabular}{|c|c|c|c|c|c|c|}
\hline \multirow{2}{*}{$\begin{array}{l}\text { PERFECT Uretsky et } \\
\text { al. } 2016 \text { [28] }\end{array}$} & $\begin{array}{l}\text { Method of randomization } \\
\text { not reported }\end{array}$ & Not reported & No & Not reported & Yes & \multirow[t]{2}{*}{ Low risk } \\
\hline & Low risk & Unclear & High risk & Unclear & High risk & \\
\hline \multirow{2}{*}{$\begin{array}{l}\text { CRESCENT-II } \\
\text { Lubbers et al. } 2017 \\
\text { [13] }\end{array}$} & $\begin{array}{l}\text { Method of randomization } \\
\text { not reported }\end{array}$ & Not reported & No & No & Not reported & \multirow[t]{2}{*}{ Low risk } \\
\hline & Low risk & Unclear & High risk & High risk & Unclear & \\
\hline
\end{tabular}

\section{TABLE 5: Cochrane risk of bias for quality assessment}

CTA, computed tomography angiogram.

Heterogeneity

We used $\mathrm{I}^{2}$ statistics to calculate the heterogeneity. $\mathrm{I}^{2}>50 \%$ was considered substantial heterogeneity, as explained in the Cochrane Handbook for Systematic Reviews [29]. We performed a sensitivity analysis for considerable heterogeneity.

\section{Results}

We included 16 RCTs with 21,210 patients (10,937 in the CCTA arm and 10,273 in the ST arm). Patients in the ST arm were older than those in the CCTA arm (57.9 \pm 9.8 years vs. $57.4 \pm 10$ years, respectively; $\mathrm{P}=$ .0002 ) and had more hyperlipidemia ( $45.62 \%$ vs. $43.18 \%$, respectively; $\mathrm{P}=.0004)$. There was no difference in baseline body mass index, hypertension, diabetes, smoking status, and baseline use of aspirin. Three studies used ST without imaging for a total of 1,110 patients (595 in the CCTA arm and 515 in the ST without imaging arm) $[17,22,26]$.

Primary endpoints were all-cause mortality and new myocardial infarction (MI) during the follow-up period. Secondary endpoints included ICA after ST, true positive ICA, revascularizations, new unstable anginas, emergency room (ER) visits or hospital admissions during the follow-up period, follow-up tests, complications (stroke, bleeding, anaphylaxis, or renal failure) attributed to CCTA compared to ST, direct discharges from ER, ER cost and total cost, and radiation dose. The results are summarized in Table 6.

\begin{tabular}{|c|c|c|c|c|c|c|}
\hline Outcome & ССТА & ST & Effect Estimate & Confidence Interval & P-value & $\mathbf{I}^{2}$ \\
\hline \multicolumn{7}{|l|}{ Primary Outcomes } \\
\hline All-Cause Mortality & 103 & 110 & 0.93 & $0.71-1.21$ & .58 & $0 \%$ \\
\hline ST with Imaging & 100 & 108 & 0.92 & $0.70-1.21$ & .55 & $0 \%$ \\
\hline ST without Imaging & 3 & 2 & 1.26 & $0.21-7.71$ & .8 & $0 \%$ \\
\hline All-Cause Mortality & 103 & 110 & 0.93 & $0.71-1.21$ & .58 & $0 \%$ \\
\hline Acute Chest Pain & 9 & 12 & 0.75 & $0.30-1.89$ & .54 & $0 \%$ \\
\hline Stable Chest Pain & 103 & 110 & 0.95 & $0.71-1.25$ & .7 & $0 \%$ \\
\hline New Myocardial Infarction & 115 & 156 & 0.71 & $0.56-0.91$ & .006 & $0 \%$ \\
\hline ST with Imaging & 108 & 151 & 0.7 & $0.54-0.89$ & .004 & $0 \%$ \\
\hline ST without Imaging & 7 & 5 & 1.14 & $0.35-3.75$ & .83 & $0 \%$ \\
\hline New Myocardial Infarction & 115 & 156 & 0.71 & $0.56-0.91$ & .006 & $0 \%$ \\
\hline Acute Chest Pain & 35 & 36 & 0.88 & $0.54-1.44$ & .61 & $0 \%$ \\
\hline Stable Chest Pain & 80 & 20 & 0.66 & $0.5-0.88$ & .004 & $0 \%$ \\
\hline \multicolumn{7}{|l|}{ Secondary Outcomes } \\
\hline Cumulative ICA & 1,044 & 701 & 1.41 & $1.28-1.55$ & $<.00001$ & $1 \%$ \\
\hline ST with Imaging & 948 & 637 & 1.37 & $1.21-1.55$ & $<.00001$ & $11 \%$ \\
\hline
\end{tabular}




\section{Cureus}

\begin{tabular}{|c|c|c|c|c|c|c|}
\hline ST without Imaging & 96 & 64 & 1.39 & $1.04-1.85$ & .02 & $0 \%$ \\
\hline Cumulative ICA & 1,044 & 701 & 1.41 & $1.28-1.55$ & $<.00001$ & $1 \%$ \\
\hline Acute Chest Pain & 311 & 205 & 1.35 & 1.13-1.62 & .001 & $8 \%$ \\
\hline Stable Chest Pain & 733 & 496 & 1.44 & $1.30-1.61$ & $<.00001$ & $0 \%$ \\
\hline True Positive ICA & 629 & 270 & 2.85 & $2.28-3.56$ & $<.00001$ & $0 \%$ \\
\hline ST with Imaging & 565 & 246 & 2.84 & 2.25-3.59 & $<.00001$ & $0 \%$ \\
\hline ST without Imaging & 64 & 24 & 4.67 & $1.15-18.91$ & .03 & $48 \%$ \\
\hline True Positive ICA & 629 & 270 & 2.85 & $2.28-3.56$ & $<.00001$ & $0 \%$ \\
\hline Acute Chest Pain & 117 & 41 & 3.2 & $1.83-5.60$ & $<.001$ & $0 \%$ \\
\hline Stable Chest Pain & 512 & 229 & 2.79 & 2.19-3.55 & $<.00001$ & $0 \%$ \\
\hline Cumulative Revascularization & 789 & 472 & 1.84 & $1.44-2.35$ & $<.00001$ & $53 \%$ \\
\hline ST with Imaging & 737 & 450 & 1.77 & $1.34-2.33$ & $<.0001$ & $60 \%$ \\
\hline ST without Imaging & 52 & 22 & 2.36 & $1.40-3.98$ & .001 & $0 \%$ \\
\hline Cumulative Revascularization & 789 & 472 & 1.84 & $1.44-2.35$ & $<.00001$ & $53 \%$ \\
\hline Acute Chest Pain & 175 & 82 & 1.95 & $1.42-2.69$ & $<.0001$ & $17 \%$ \\
\hline Stable Chest Pain & 614 & 390 & 1.7 & $1.16-2.51$ & .007 & $77 \%$ \\
\hline New Unstable Anginas & 257 & 198 & 1.18 & 0.99-1.41 & .06 & $0 \%$ \\
\hline ST with Imaging & 245 & 191 & 1.18 & $0.98-1.40$ & .07 & $0 \%$ \\
\hline ST without Imaging & 12 & 7 & 1.09 & $0.20-5.92$ & .92 & $49 \%$ \\
\hline New Unstable Anginas & 257 & 198 & 1.18 & $0.99-1.41$ & .06 & $0 \%$ \\
\hline Acute Chest Pain & 118 & 84 & 1.15 & $0.90-1.48$ & .27 & $0 \%$ \\
\hline Stable Chest Pain & 139 & 114 & 1.21 & 0.93-1.58 & .15 & $4 \%$ \\
\hline ER visits or hospital admissions & 570 & 616 & 0.75 & $0.60-0.94$ & .01 & $63 \%$ \\
\hline ST with Imaging & 554 & 551 & 0.92 & 0.83-1.02 & .11 & $0 \%$ \\
\hline ST without Imaging & 16 & 65 & 0.27 & $0.15-0.48$ & $<.0001$ & $27 \%$ \\
\hline ER visits or hospital admissions & 570 & 616 & 0.75 & $0.60-0.94$ & .01 & $63 \%$ \\
\hline Acute Chest Pain & 300 & 289 & 0.86 & $0.72-1.04$ & .11 & $22 \%$ \\
\hline Stable Chest Pain & 270 & 327 & 0.5 & $0.21-1.23$ & .13 & $86 \%$ \\
\hline Cumulative Follow up Testing & 242 & 342 & 0.45 & $0.22-0.90$ & .02 & $86 \%$ \\
\hline ST with Imaging & 159 & 197 & 0.43 & $0.16-1.14$ & .09 & $86 \%$ \\
\hline ST without Imaging & 83 & 145 & 0.39 & $0.28-0.56$ & $<.00001$ & $0 \%$ \\
\hline Cumulative Follow up Testing & 242 & 342 & 0.45 & $0.22-0.90$ & .02 & $86 \%$ \\
\hline Acute Chest Pain & 166 & 165 & 0.83 & $0.44-1.55$ & .56 & $70 \%$ \\
\hline Stable Chest Pain & 76 & 177 & 0.17 & $0.04-0.77$ & .02 & $80 \%$ \\
\hline Procedural Complications ${ }^{\star}$ & 7 & 7 & 0.98 & $0.35-2.74$ & .96 & $0 \%$ \\
\hline Direct ER Discharges & 936 & 421 & 1.45 & $0.63-3.30$ & .38 & $94 \%$ \\
\hline Cost in ER & - & - & -4.68 & $(-10.38)-(1.01)$ & .11 & $100 \%$ \\
\hline Total Downstream Cost & - & - & -0.01 & $(-0.17)-(0.14)$ & .85 & $45 \%$ \\
\hline Cumulative Radiation Dose & $7.3 \pm 6.6$ & $2.6 \pm 6.5$ & 0.47 & $0.08-0.86$ & .02 & $97 \%$ \\
\hline
\end{tabular}




\section{Cureus}

\section{TABLE 6: Outcomes}

* Procedural complications include stroke, bleeding, anaphylaxis, or renal failure

Abbreviations: ER, emergency room; ICA, invasive coronary angiography; ST, stress testing.

\section{Primary Endpoints}

All-cause mortality: There was no difference in all-cause mortality (103 vs. $110 ; \mathrm{RR}=0.93, \mathrm{CI}=0.71-1.21 ; \mathrm{P}$ $\left.=.58, \mathrm{I}^{2}=0 \%\right)$. The subgroup analyses for ST with imaging $\left(\mathrm{RR}=0.92, \mathrm{CI}=0.70-1.21 ; \mathrm{P}=.55, \mathrm{I}^{2}=0 \%\right), \mathrm{ST}$ without imaging $\left(\mathrm{RR}=1.26, \mathrm{CI}=0.21-7.71 ; \mathrm{P}=.80, \mathrm{I}^{2}=0 \%\right), \mathrm{ACP}\left(\mathrm{RR}=0.75, \mathrm{CI}=0.30-1.89 ; \mathrm{P}=.54, \mathrm{I}^{2}=0 \%\right)$ and SCP $\left(\mathrm{RR}=0.95, \mathrm{CI}=0.71-1.25 ; \mathrm{P}=.70, \mathrm{I}^{2}=0 \%\right.$ ) found no differences (Figure $3 A$ and $\left.3 \mathrm{~B}\right)$ [13-28].

A

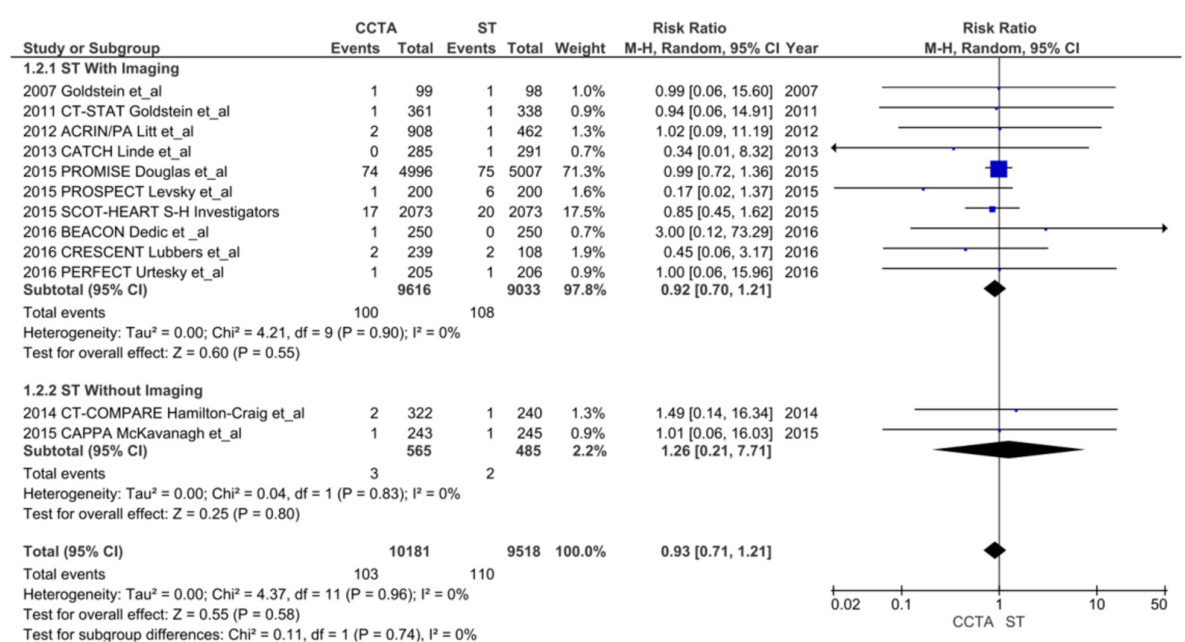

B

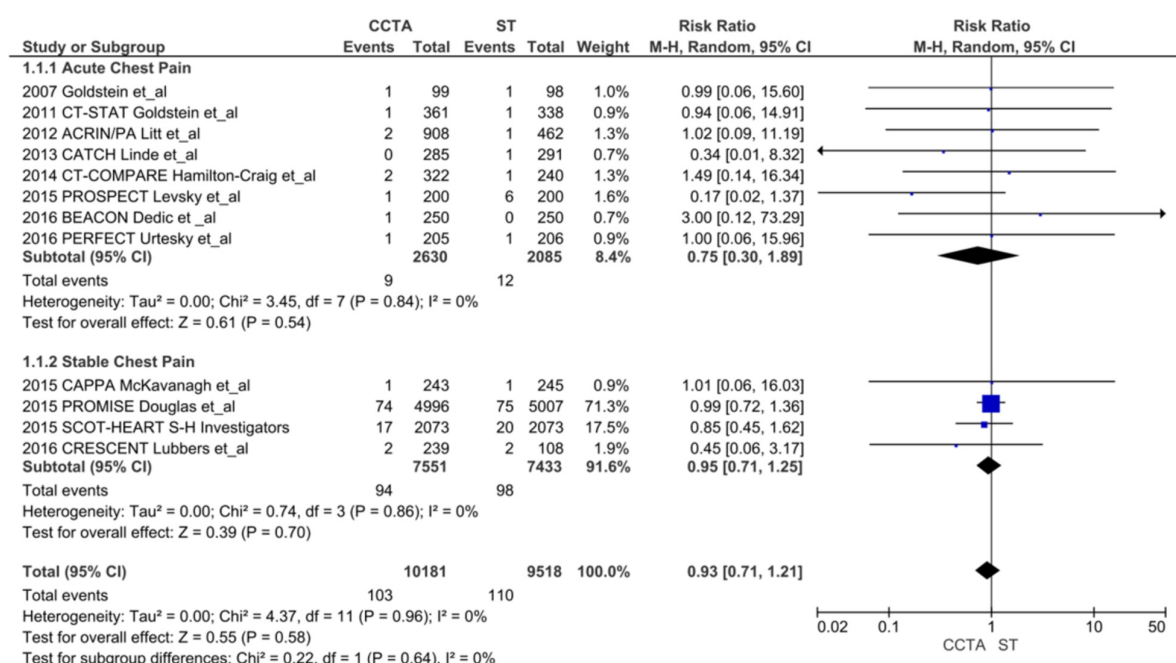

FIGURE 3: All-cause mortality

CCTA, coronary computed tomography angiography; ST, stress testing.

A. ST with imaging vs. ST without imaging [13-28]

B. Acute chest pain (ACP) vs. stable chest pain (SCP) [13-28] 


\section{Cureus}

New MI during follow-up period: A significant reduction in the incidence of future MI was noticed in the CCTA arm (115 vs. $\left.156 ; \mathrm{RR}=0.71, \mathrm{CI}=0.56-0.91 ; \mathrm{P}<.006, \mathrm{I}^{2}=0 \%\right)$; this was mainly noted as a reduction in $\mathrm{MI}$ in the SCP subgroup patients ( $80 \mathrm{vs.} .120 ; \mathrm{RR}=0.66, \mathrm{CI}=0.50-0.88 ; \mathrm{P}=.004, \mathrm{I}^{2}=0 \%$ ) compared to the ACP subgroup that showed no difference ( $35 \mathrm{vs.} 36 ; \mathrm{RR}=0.88, \mathrm{CI}=0.54-1.44 ; \mathrm{P}=.61, \mathrm{I}^{2}=0 \%$ ). The CCTA arm also had significantly reduced MIs compared to ST with imaging $\left(\mathrm{RR}=0.70, \mathrm{CI}=0.54-0.89 ; \mathrm{P}=.004, \mathrm{I}^{2}=0 \%\right)$ with no difference compared to ST without imaging $\left(\mathrm{RR}=1.14, \mathrm{CI}=0.35-3.75 ; \mathrm{P}=.83, \mathrm{I}^{2}=0 \%\right.$; Figure $4 \mathrm{~A}$ and $\left.4 \mathrm{~B}\right)$ [13-28]. The NNT after CCTA to prevent one MI was 204 and NNT after ICA to prevent one MI was nine.

A

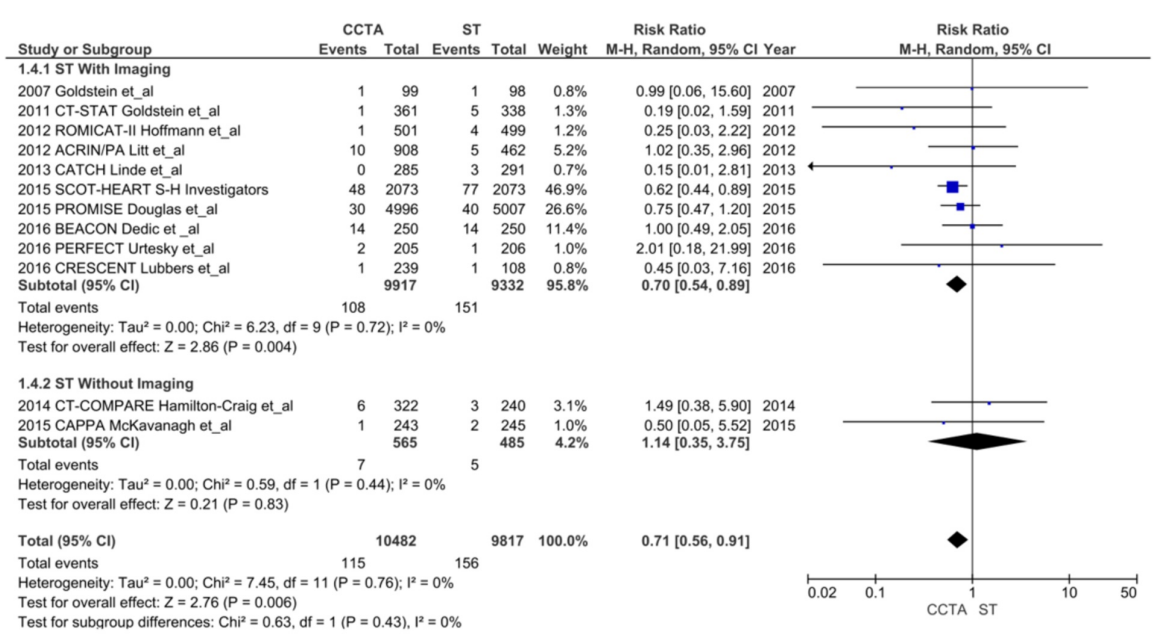

B

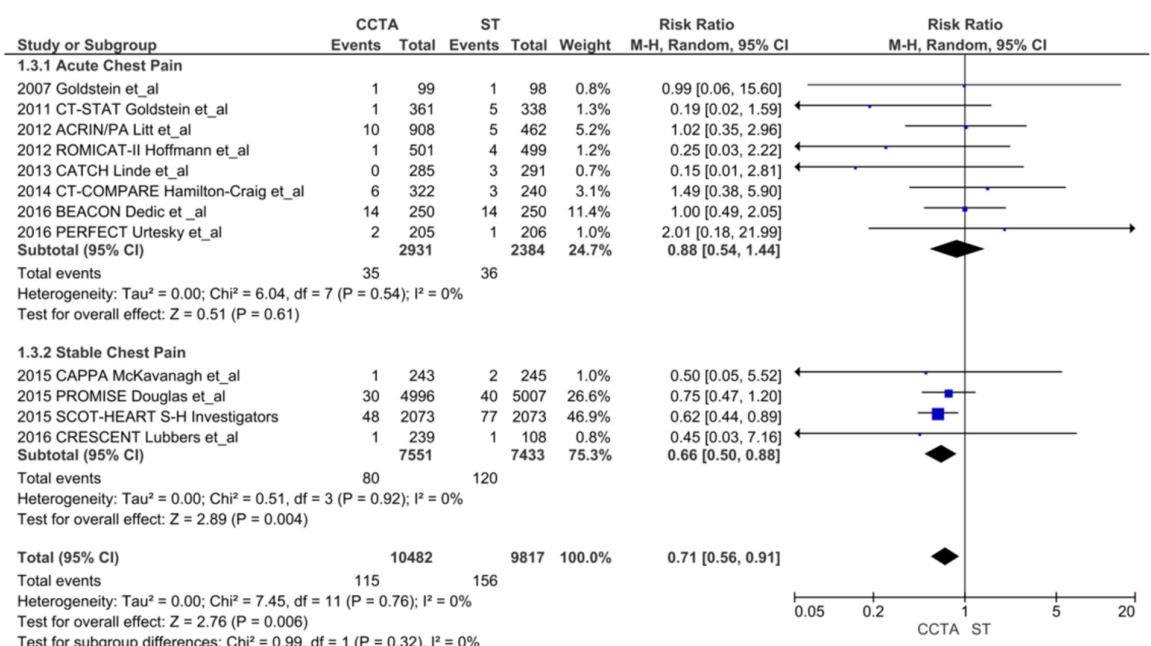

\section{FIGURE 4: New myocardial infarction during the follow-up period}

CCTA, coronary computed tomography angiography; ST, stress testing; $\mathrm{Cl}$, confidence interval.

A. ST with imaging vs. without imaging [13-28]

B. Acute chest pain vs. stable chest pain [13-28]

\section{Secondary Endpoints}

ICA after ST: The CCTA arm had significantly increased ICA (1,044 vs. 701; RR =1.41, CI =1.28-1.55; P < 


\section{Cureus}

$\left..00001, \mathrm{I}^{2}=1 \%\right)$. Both the ACP (311 vs. $205 ; \mathrm{RR}=1.35, \mathrm{CI}=1.13-1.62 ; \mathrm{P}=.001, \mathrm{I}^{2}=8 \%$ ) and SCP (733 vs. 496; $\left.\mathrm{RR}=1.44, \mathrm{CI}=1.30-1.61 ; \mathrm{P}<.00001, \mathrm{I}^{2}=0 \%\right)$ subgroups had more ICA post-CCTA. ICA was common after CCTA compared to ST with imaging $\left(\mathrm{RR}=1.37, \mathrm{CI}=1.21-1.55 ; \mathrm{P}<.00001, \mathrm{I}^{2}=11 \%\right)$ and without imaging ( $\mathrm{RR}$ $=1.37, \mathrm{CI}=1.21-1.55 ; \mathrm{P}<.00001, \mathrm{I}^{2}=11 \%$; Figure $5 \mathrm{~A}$ and $\left.5 \mathrm{~B}\right)[13-28]$. We did not include ICA from the SCOTHEART study as they only reported new or canceled ICA in their manuscript and appendix [23].

A

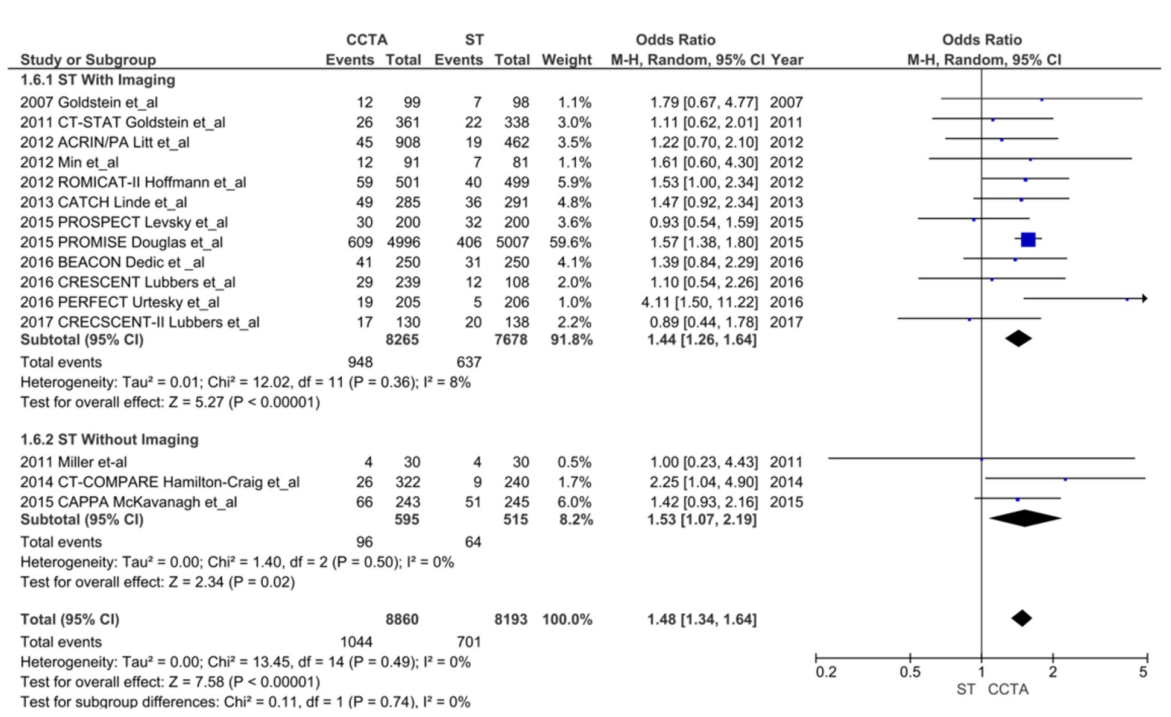

B

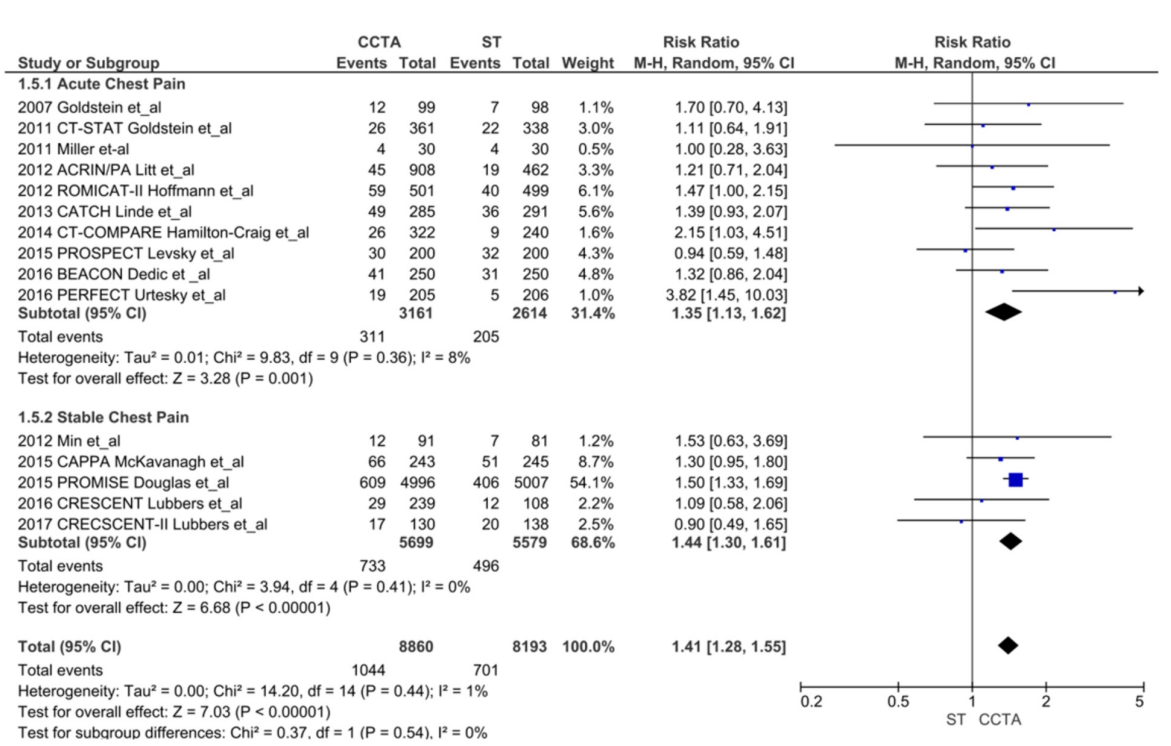

\section{FIGURE 5: Invasive coronary angiograms}

CCTA, coronary computed tomography angiography; ST, stress testing.

A. ST with imaging vs. without imaging [13-28]

B. Acute chest pain vs. stable chest pain [13-28]

True positive ICA: CCTA lead to a significantly higher diagnosis of obstructive CAD (stenosis $\geqslant 50 \%$ ) compared to ST (629/883 after CCTA vs. 270/587 after ST; OR = 2.85, CI = 2.28-3.56; $\left.\mathrm{P}<0.00001, \mathrm{I}^{2}=0 \%\right)$. 


\section{Cureus}

This finding was consistent in both the ACP $\left(\mathrm{OR}=3.20, \mathrm{CI}=1.83-5.60 ; \mathrm{P}<.001, \mathrm{I}^{2}=0 \%\right)$ and $\mathrm{SCP}(\mathrm{OR}=2.79$, $\left.\mathrm{CI}=2.19-3.55 ; \mathrm{P}<.00001, \mathrm{I}^{2}=0 \%\right)$ subgroups and in $\mathrm{ST}$ with imaging $(\mathrm{OR}=2.84, \mathrm{CI}=2.25-3.59 ; \mathrm{P}<.00001$, $\left.\mathrm{I}^{2}=0 \%\right)$ and without imaging $\left(\mathrm{OR}=4.67, \mathrm{CI}=1.15-18.91 ; \mathrm{P}=.03, \mathrm{I}^{2}=48 \%\right.$; Figure $6 \mathrm{~A}$ and $\left.6 \mathrm{~B}\right)[13-28]$.

\section{A}

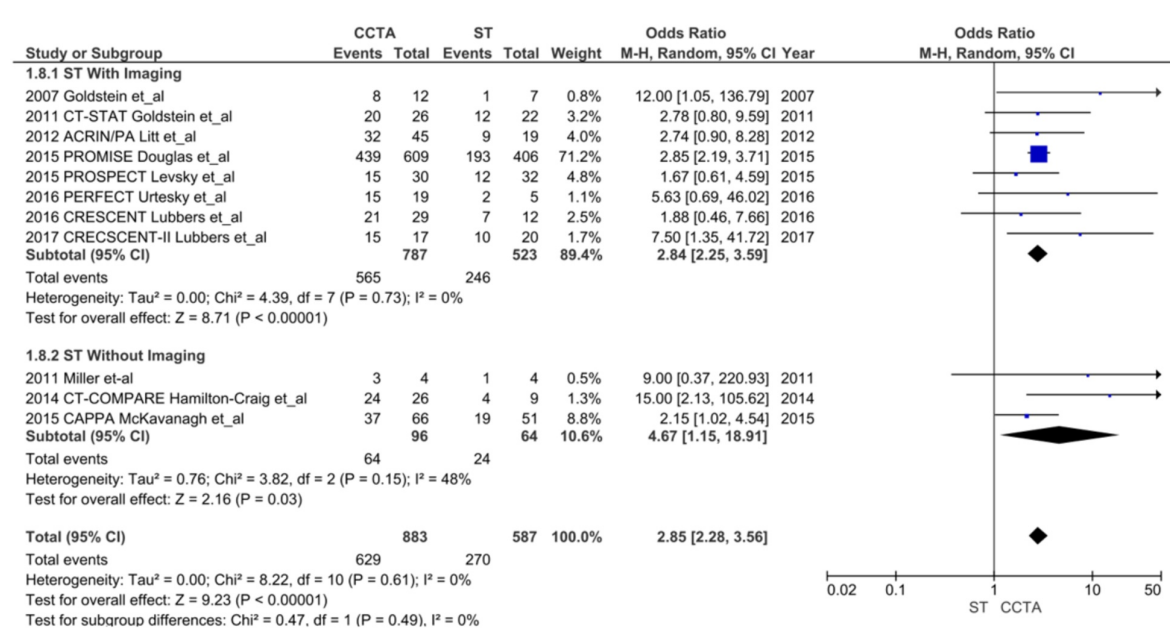

B

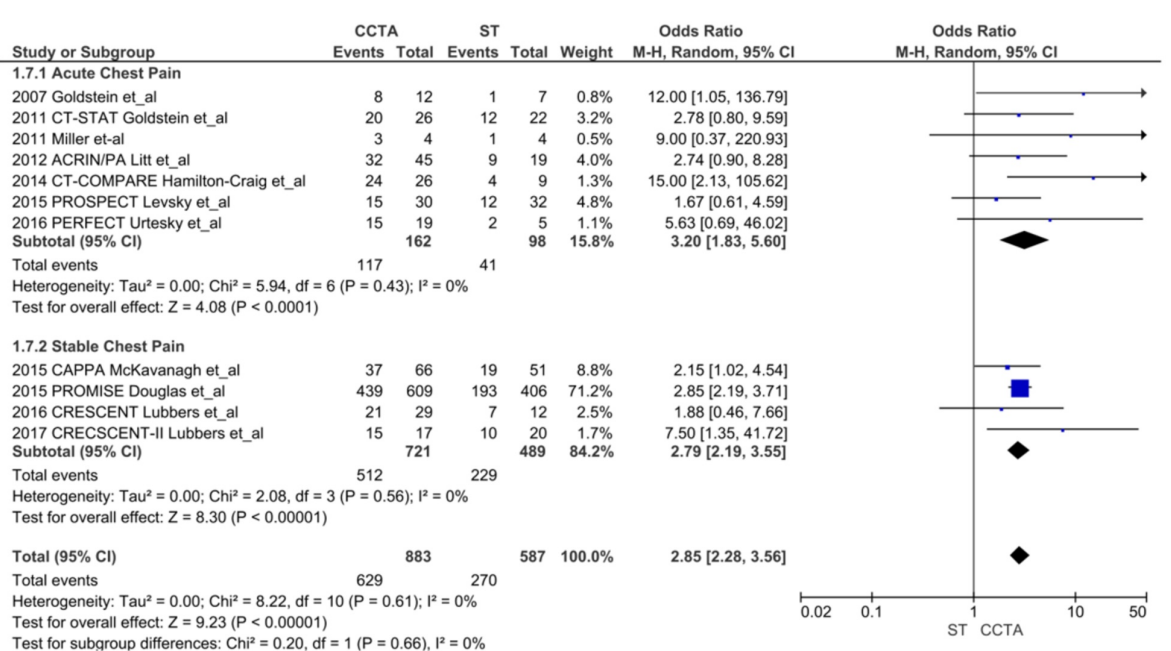

FIGURE 6: True positive invasive coronary angiograms

CCTA, coronary computed tomography angiography; ST, stress testing.

A. ST with imaging vs. without imaging [13-28]

B. Acute chest pain vs. stable chest pain [13-28]

Revascularization: Revascularization (percutaneous coronary intervention and coronary artery bypass grafting) was significantly higher after CCTA (789 vs. 472; OR = 1.84, CI = 1.44-2.35; P <.00001, $\mathrm{I}^{2}=53 \%$ ). Of note, $\mathrm{I}^{2}$ was reduced to $0 \%$ with exclusion of the SCOT-HEART trial and without affecting significance [24]. This trend was consistent on subgroup analysis of ST with imaging $\left(\mathrm{RR}=1.77, \mathrm{CI}=1.34-2.33 ; \mathrm{P}<.00001, \mathrm{I}^{2}=\right.$ $60 \%)$, ST without imaging $\left(\mathrm{RR}=2.36, \mathrm{CI}=1.40-3.98 ; \mathrm{P}=.001, \mathrm{I}^{2}=0 \%\right)$, ACP (175 vs. $82 ; \mathrm{OR}=1.95, \mathrm{CI}=1.42-$ 2.69; $\left.\mathrm{P}<.0001, \mathrm{I}^{2}=17 \%\right)$, and $\mathrm{SCP}\left(614\right.$ vs. $390 ; \mathrm{OR}=1.70, \mathrm{CI}=1.16-2.51 ; \mathrm{P}=.007, \mathrm{I}^{2}=77 \%$ and $0 \%$ without inclusion of the SCOT-HEART trial [23]. 


\section{Cureus}

New unstable angina: There was no difference in new unstable anginas in the CCTA group vs. ST group (257 vs. $198 ; \mathrm{RR}=1.18, \mathrm{CI}=0.99-1.41 ; \mathrm{P}=.06, \mathrm{I}^{2}=0 \%$ ). A similar trend was seen on subgroup analysis of $\mathrm{ACP}$ (118 vs. $\left.84 ; \mathrm{RR}=1.15, \mathrm{CI}=0.90-1.48 ; \mathrm{P}=.27, \mathrm{I}^{2}=0 \%\right)$, $\mathrm{SCP}\left(139\right.$ vs. $114 ; \mathrm{RR}=1.21, \mathrm{CI}=0.93-1.58 ; \mathrm{P}=.15, \mathrm{I}^{2}=$ $4 \%)$, ST with imaging $\left(\mathrm{RR}=1.18, \mathrm{CI}=0.98-1.40 ; \mathrm{P}=.07, \mathrm{I}^{2}=0 \%\right)$ and $\mathrm{ST}$ without imaging, $(\mathrm{RR}=1.09, \mathrm{CI}=$ $\left.0.20-5.92 ; \mathrm{P}=.92, \mathrm{I}^{2}=49 \%\right)$.

ER visits and/or hospital admissions during the follow-up period: ER visits and/or hospital admissions were reduced significantly in the CCTA arm (570 vs. 616 ; $\mathrm{RR}=0.75, \mathrm{CI}=0.60-0.94 ; \mathrm{P}=.01, \mathrm{I}^{2}=63 \%$ ). $\mathrm{I}^{2}$ was reduced to $16 \%$ without the CAPPA trial, but the results became statistically insignificant. The subgroup analysis of ACP and SCP and ST with imaging revealed no difference between CCTA and ST, though there were significantly reduced ER visits or hospital admissions in the CCTA arm compared to ST without imaging $\left(\mathrm{RR}=0.27, \mathrm{CI}=0.15-0.48 ; \mathrm{P}<.0001 ; \mathrm{I}^{2}=27 \%\right.$; Figure $7 \mathrm{~A}$ and $\left.7 \mathrm{~B}\right)[13-28]$.

A

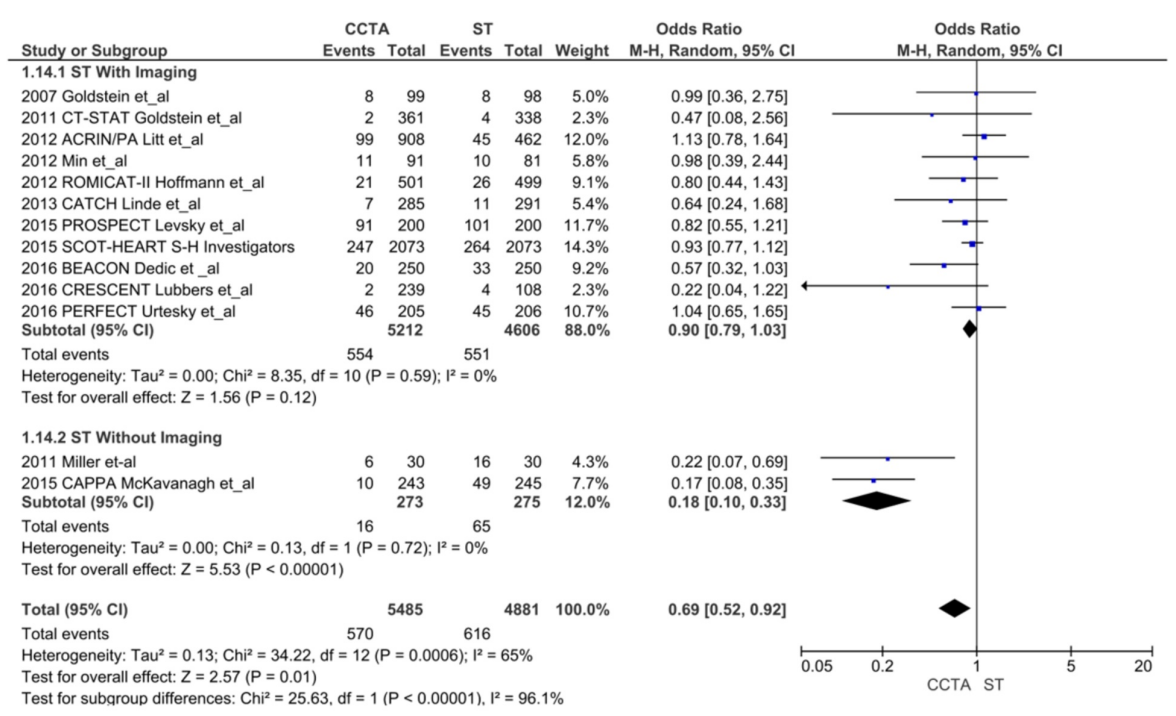

B

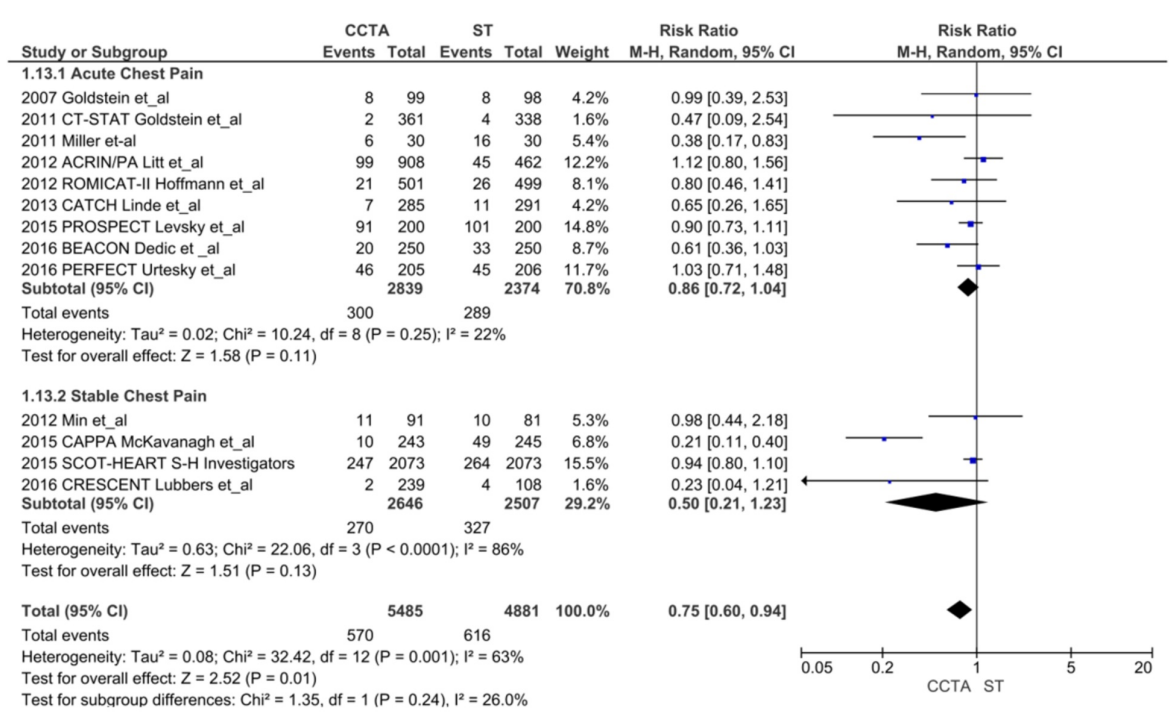

FIGURE 7: Emergency room visits or hospital admissions during the follow-up period

CCTA, coronary computed tomography angiography; ST, stress testing. 


\section{Cureus}

A. ST with imaging vs. without imaging [13-28]

B. Acute chest pain vs. stable chest pain [13-28]

Follow-up tests: Patients in the CCTA arm had a significant reduction in downstream testing (242 vs. 342; $\left.\mathrm{OR}=0.45, \mathrm{CI}=0.22-0.90 ; \mathrm{P}=.02, \mathrm{I}^{2}=86 \%\right)$; sensitivity analysis did not reduce the heterogeneity. The ST with imaging subgroup $\left(\mathrm{RR}=0.43, \mathrm{CI}=0.16-1.14 ; \mathrm{P}=.09, \mathrm{I}^{2}=86 \%\right)$ and $\mathrm{ACP}$ subgroup $(\mathrm{RR}=0.83, \mathrm{CI}=0.44$ $\left.1.55 ; \mathrm{P}=.56, \mathrm{I}^{2}=70 \%\right)$ showed no difference in follow-up testing. ST without imaging $(\mathrm{RR}=0.39, \mathrm{CI}=0.28-$ $\left.0.56 ; \mathrm{P}<.00001, \mathrm{I}^{2}=0 \%\right)$ and the SCP subgroup $\left(\mathrm{RR}=0.17, \mathrm{CI}=0.04-0.77 ; \mathrm{P}=.02, \mathrm{I}^{2}=80 \%\right)$ had a significant reduction in follow-up testing after CCTA (Figure $8 \mathrm{~A}$ and $8 \mathrm{~B}$ ) [13-28].

A

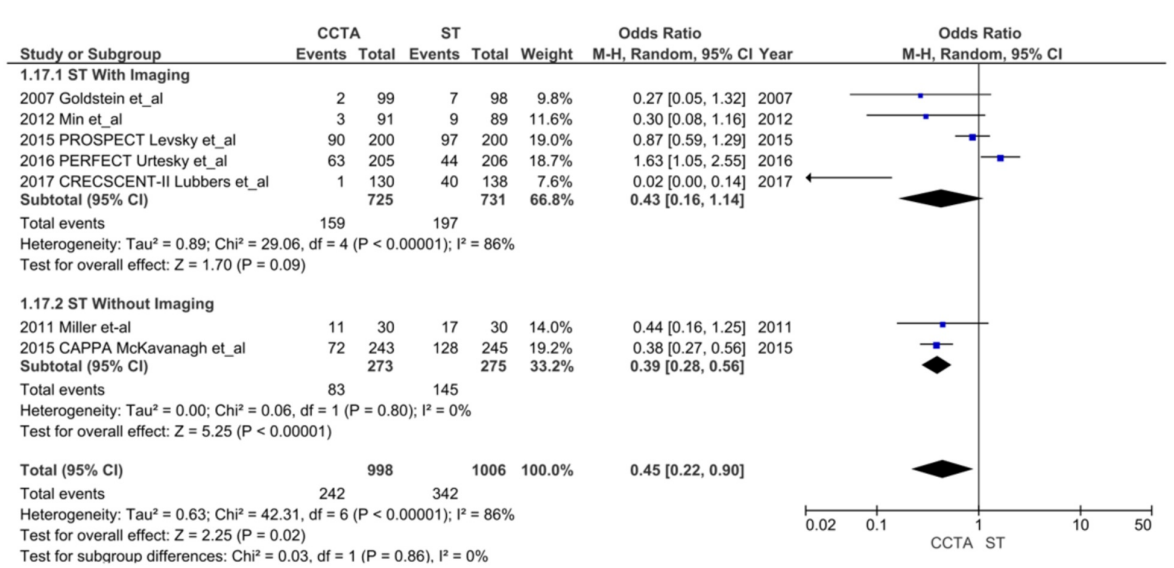

B

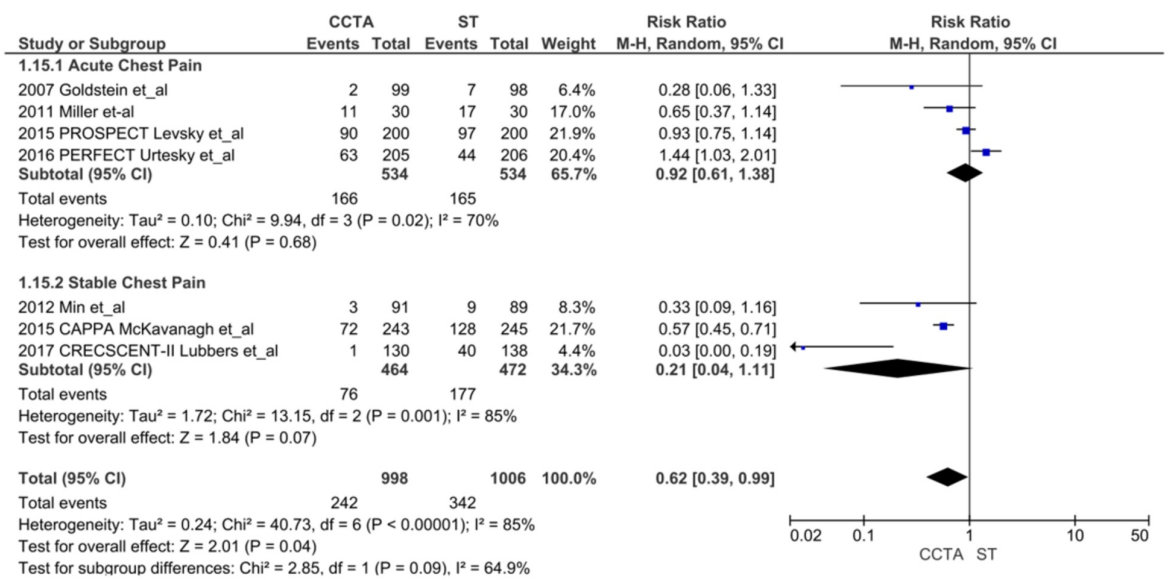

\section{FIGURE 8: Follow-up tests}

CCTA, coronary computed tomography angiography; ST, stress testing.

A. ST with imaging vs. without imaging [13-28]

B. Acute chest pain vs. stable chest pain [13-28]

Complications associated with CCTA vs. ST: Only four studies reported serious complications attributed to investigation modalities used in the trials. We did not identify any difference between the two arms (7 vs. 7 ; $\left.\mathrm{RR}=0.98, \mathrm{CI}=0.35-2.74 ; \mathrm{P}=.96, \mathrm{I}^{2}=0 \%\right)$. 
Direct discharge from the ER: Five studies reported direct ER discharges without admission to the hospital $\left(\right.$ CCTA arm $=936$ vs. $\mathrm{ST}$ arm $\left.=421 ; \mathrm{OR}=1.45, \mathrm{CI}=0.63-3.30 ; \mathrm{P}=.38, \mathrm{I}^{2}=94 \%\right)$; sensitivity analysis did not reduce heterogeneity.

Cost analysis: Eight studies reported cost, but only five studies were usable as these reported mean cost and standard deviation.

Three studies reported the ER costs. There was a trend towards a decrease in ER costs in the CCTA arm (standardized mean difference $[\mathrm{SMD}]=-4.68, \mathrm{CI}=-10.38$ to $1.01 ; \mathrm{P}=.11, \mathrm{I}^{2}=100 \%$ ). Sensitivity analysis, without the CT-COMPARE trial, reduced the heterogeneity to $0 \%$ and the results became statistically significant $\left(\mathrm{SMD}=-0.38, \mathrm{CI}=-0.51\right.$ to $-0.26 ; \mathrm{P}=.00001, \mathrm{I}^{2}=0 \%$ ).

Five studies reported the total cost. There was no difference between the two arms $(\mathrm{SMD}=-0.64, \mathrm{CI}=-1.75$ to $\left.0.46 ; \mathrm{P}=.25, \mathrm{I}^{2}=99 \%\right)$. Sensitivity analysis without the CT-COMPARE reduced the heterogeneity to $45 \%$; however, the results remained statistically insignificant. The subgroup analysis for the cost in the United States and cost elsewhere also had significant heterogeneity with no difference between the subgroups (chisquared $=0.15$, degrees of freedom $=1, \mathrm{P}=.69, \mathrm{I}^{2}=0 \%$ ).

Radiation dose: Four studies reported the cumulative radiation exposure usable for our analysis. The CCTA arm had significantly higher radiation exposure $\left(\mathrm{SMD}=0.47, \mathrm{CI}=0.08-0.86 ; \mathrm{P}=.02, \mathrm{I}^{2}=97 \%\right)$. Sensitivity analysis failed to reduce the heterogeneity.

\section{Discussion}

Our meta-analysis of 21,210 patients comparing CCTA to ST demonstrated a significant reduction in the primary endpoint of MIs in the CCTA group without any difference in mortality. The reduction in MI was secondary to a significantly reduced number of events in the SCP group. The reduction in MIs is likely due to the early diagnosis of obstructive $\mathrm{CAD}$ and subsequent early initiation of aggressive medical management and revascularizations. Recently published five-year outcomes of the SCOT-HEART trial, which enrolled patients with SCP, also showed a significant reduction in MIs over five years [30]. This discrepancy in downstream MIs between the ACP and SCP group calls for a novel assessment strategy to risk-stratify ACP patients who present to the ER regarding invasive versus conservative management. The lack of mortality benefit in our analysis may not be evident because of the short follow-up times of the individual studies (four weeks to 25 months) compared to the five-year outcomes of the SCOT-HEART trial which showed a significant reduction in mortality from coronary heart disease or nonfatal MI than standard care alone [30].

This analysis also showed increased ICA and revascularizations, which also lead to significantly reduced MIs (NNT of nine to prevent one MI for each ICA). This early difference in ICA and revascularization may be lost after an extended follow-up as suggested by the five-year outcomes of the SCOT-HEART study [23]. This indicates that CCTA use leads to early diagnosis of CAD and subsequent early intervention compared to the ST, where patients eventually needed ICA and revascularization at the cost of increased MIs and mortality. Due to the high sensitivity of CCTA (approximately 99\%), a negative CCTA may reduce further testing whereas a positive CCTA leads to additional invasive procedures. In our analysis, there were significantly more ICA, true positive ICA, and revascularizations, with significantly reduced follow-up tests. The use of CCTA leads to a higher number of invasive procedures, including revascularization, ultimately leading to higher costs overall.

After the initial randomization and workup with either CCTA or ST, ER visits and rehospitalizations were significantly reduced in the CCTA arm; this differs from a previously published meta-analysis that showed no difference in ER visits and rehospitalizations [7-11]. A limitation of our analysis was the presence of substantial heterogeneity, making it difficult to generalize the results. The sensitivity analysis reduced heterogeneity with a trend towards reduced ER visits or rehospitalizations in the CCTA arm. Reduction in ER visits and rehospitalizations is promising, as earlier studies found that the reduced MIs after CCTA group was offset by increased future rehospitalizations and downstream costs.

The increased rates of angiographically confirmed CAD post-CCTA is another significant finding that suggests that CCTA has a better positive predictive value than ST (with or without imaging) to identify obstructive $\mathrm{CAD}$ at a time when current guidelines do not support the routine use of CCTA in intermediaterisk patients. Although our analysis showed an increasing trend towards unstable anginas in the CCTA arm, we hypothesize that this trend is likely the consequence of higher rates of revascularization in the CCTA group.

The cost analysis had substantial heterogeneity for both ER visits and downstream costs. The trials included in our analysis were conducted in different countries with different healthcare systems and cost structures [13-28]. In our analysis, even though a trend towards decreased ER costs was seen in the CCTA arm, there was no clear advantage of total downstream cost to either imaging strategy. In the absence of any significant mortality benefit, it is reassuring that whichever approach the provider offers will not adversely affect the patient. CCTA was associated with significantly higher cumulative radiation exposure; however, 


\section{Limitations}

Our study had several significant limitations. First, a lack of long-term follow-up in the individual RCTs $(\leqslant 25$ months) that may not include events, hospitalizations, and revascularizations beyond 25 months would magnify the risks of ICA and revascularization and obscure potential long-term benefits. This may be true for ACP trials as short follow-up may have masked the advantage for either arm. Also, some outcomes were not reported by most studies, leading to substantial heterogeneity that persisted even after sensitivity analysis. In addition, we were unable to estimate radiation exposure from all studies between the two groups since they reported data in a variable form. Also, only three studies used ST without imaging, and the other studies used a combination of imaging and non-imaging ST; this leads to substantial overlap between the groups and has a risk to introduce bias in our results. Finally, these trials, although relatively modern, did not utilize high-sensitivity cardiac troponin tests. Their hypotheses must be tested again with the advent of these tests.

\section{Conclusions}

Our analysis is the largest to date of 16 RCTs and found a significant reduction in post-CCTA MIs with increased ICA and revascularizations. In the future, more RCTs are needed utilizing scoring methods to identify more robust downstream investigations, cost analysis, and radiation exposure.

\section{Additional Information \\ Disclosures}

Human subjects: All authors have confirmed that this study did not involve human participants or tissue. Animal subjects: All authors have confirmed that this study did not involve animal subjects or tissue. Conflicts of interest: In compliance with the ICMJE uniform disclosure form, all authors declare the following: Payment/services info: All authors have declared that no financial support was received from any organization for the submitted work. Financial relationships: All authors have declared that they have no financial relationships at present or within the previous three years with any organizations that might have an interest in the submitted work. Other relationships: All authors have declared that there are no other relationships or activities that could appear to have influenced the submitted work.

\section{References}

1. Shaw LJ, Min JK, Hachamovitch R, et al.: Cardiovascular imaging research at the crossroads . JACC Cardiovasc Imaging. 2010, 3:316-324. 10.1016/j.jcmg.2009.11.010

2. Sekhri N, Feder GS, Junghans C, Hemingway H, Timmis AD: How effective are rapid access chest pain clinics? Prognosis of incident angina and non-cardiac chest pain in 8762 consecutive patients. Heart. 2007, 93:458-463. 10.1136/hrt.2006.090894

3. Miller JM, Rochitte CE, Dewey M, et al.: Diagnostic performance of coronary angiography by 64-row CT . N Engl J Med. 2008, 359:2324-2336. 10.1056/NEJMoa0806576

4. Budoff MJ, Dowe D, Jollis JG, et al.: Diagnostic performance of 64-multidetector row coronary computed tomographic angiography for evaluation of coronary artery stenosis in individuals without known coronary artery disease: results from the prospective multicenter ACCURACY (Assessment by Coronary Computed Tomographic Angiography of Individuals Undergoing Invasive Coronary Angiography) trial. J Am Coll Cardiol. 2008, 52:1724-1732. 10.1016/j.jacc.2008.07.031

5. Abdulla J, Abildstrom SZ, Gotzsche O, Christensen E, Kober L, Torp-Pedersen C: 64-multislice detector computed tomography coronary angiography as potential alternative to conventional coronary angiography: a systematic review and meta-analysis. Eur Heart J. 2007, 28:3042-3050. 10.1093/eurheartj/ehm466

6. Al-Mallah MH, Aljizeeri A, Villines TC, Srichai MB, Alsaileek A: Cardiac computed tomography in current cardiology guidelines. J Cardiovasc Comput Tomogr. 2015, 9:514-523. 10.1016/j.jcct.2015.09.003

7. D'Ascenzo F, Cerrato E, Biondi-Zoccai G, et al.: Coronary computed tomographic angiography for detection of coronary artery disease in patients presenting to the emergency department with chest pain: a metaanalysis of randomized clinical trials. Eur Heart J Cardiovasc Imaging. 2013, 14:782-789. 10.1093/ehjci/jes287

8. Hulten E, Pickett C, Bittencourt MS, Villines TC, Petrillo S, Di Carli MF, Blankstein R: Outcomes after coronary computed tomography angiography in the emergency department: a systematic review and metaanalysis of randomized, controlled trials. J Am Coll Cardiol. 2013, 61:880-892. 10.1016/j.jacc.2012.11.061

9. El-Hayek G, Benjo A, Uretsky S, et al.: Meta-analysis of coronary computed tomography angiography versus standard of care strategy for the evaluation of low risk chest pain: are randomized controlled trials and cohort studies showing the same evidence?. Int J Cardiol. 2014, 177:238-245. 10.1016/j.ijcard.2014.09.012

10. Bittencourt MS, Hulten EA, Murthy VL, Cheezum M, Rochitte CE, Di Carli MF, Blankstein R: Clinical outcomes after evaluation of stable chest pain by coronary computed tomographic angiography versus usual care: a meta-analysis. Circ Cardiovasc Imaging. 2016, 9:004419. 10.1161/CIRCIMAGING.115.004419

11. Foy AJ, Dhruva SS, Peterson B, Mandrola JM, Morgan DJ, Redberg RF: Coronary computed tomography angiography vs functional stress testing for patients with suspected coronary artery disease: a systematic review and meta-analysis. JAMA Intern Med. 2017, 177:1623-1631. 10.1001/jamainternmed.2017.4772

12. Liberati A, Altman DG, Tetzlaff J, et al.: The PRISMA statement for reporting systematic reviews and metaanalyses of studies that evaluate healthcare interventions: explanation and elaboration. BMJ. 2009, 
339:2700. 10.1136/bmj.b2700

13. Lubbers M, Coenen A, Kofflard M, et al.: Comprehensive cardiac CT with myocardial perfusion imaging versus functional testing in suspected coronary artery disease: the multicenter, randomized CRESCENT-II Trial. JACC Cardiovasc Imaging. 2018, 11:1625-1636. 10.1016/j.jcmg.2017.10.010

14. Lubbers M, Dedic A, Coenen A, et al.: Calcium imaging and selective computed tomography angiography in comparison to functional testing for suspected coronary artery disease: the multicentre, randomized CRESCENT trial. Eur Heart J. 2016, 37:1232-1243. 10.1093/eurheartj/ehv700

15. Goldstein JA, Gallagher MJ, O'Neill WW, Ross MA, O'Neil BJ, Raff GL: A randomized controlled trial of multi-slice coronary computed tomography for evaluation of acute chest pain. J Am Coll Cardiol. 2007, 49:863-871. 10.1016/j.jacc.2006.08.064

16. Goldstein JA, Chinnaiyan KM, Abidov A, et al.: The CT-STAT (Coronary Computed Tomographic Angiography for Systematic Triage of Acute Chest Pain Patients to Treatment) trial. J Am Coll Cardiol. 2011, 58:1414-1422. 10.1016/j.jacc.2011.03.068

17. Miller AH, Pepe PE, Peshock R, et al.: Is coronary computed tomography angiography a resource sparing strategy in the risk stratification and evaluation of acute chest pain? Results of a randomized controlled trial. Acad Emerg Med. 2011, 18:458-467. 10.1111/j.1553-2712.2011.01066.x

18. Hoffmann U, Truong QA, Schoenfeld DA, et al.: Coronary CT angiography versus standard evaluation in acute chest pain. N Engl J Med. 2012, 367:299-308. 10.1056/NEJMoa1201161

19. Litt HI, Gatsonis C, Snyder B, et al.: CT angiography for safe discharge of patients with possible acute coronary syndromes. N Engl J Med. 2012, 366:1393-1403. 10.1056/NEJMoa1201163

20. Min JK, Koduru S, Dunning AM, et al.: Coronary CT angiography versus myocardial perfusion imaging for near-term quality of life, cost and radiation exposure: a prospective multicenter randomized pilot trial. J Cardiovasc Comput Tomogr. 2012, 6:274-283. 10.1016/j.jcct.2012.06.002

21. Linde JJ, Kofoed KF, Sorgaard M, Kelbaek H, Jensen GB, Nielsen WB, Hove JD: Cardiac computed tomography guided treatment strategy in patients with recent acute-onset chest pain: results from the randomised, controlled trial: CArdiac cT in the treatment of acute CHest pain (CATCH). Int J Cardiol. 2013, 168:52575262. 10.1016/j.ijcard.2013.08.020

22. Hamilton-Craig C, Fifoot A, Hansen M, Pincus M, Chan J, Walters DL, Branch KR: Diagnostic performance and cost of CT angiography versus stress ECG--a randomized prospective study of suspected acute coronary syndrome chest pain in the emergency department (CT-COMPARE). Int J Cardiol. 2014, 177:867-873. 10.1016/j.ijcard.2014.10.090

23. SCOT-HEART investigators: CT coronary angiography in patients with suspected angina due to coronary heart disease (SCOT-HEART): an open-label, parallel-group, multicentre trial. Lancet. 2015, 385:2383-2391. 10.1016/S0140-6736(15)60291-4

24. Douglas PS, Hoffmann U, Patel MR, et al.: Outcomes of anatomical versus functional testing for coronary artery disease. N Engl J Med. 2015, 372:1291-1300. 10.1056/NEJMoa1415516

25. Levsky JM, Spevack DM, Travin MI, et al.: Coronary computed tomography angiography versus radionuclide myocardial perfusion imaging in patients with chest pain admitted to telemetry: a randomized trial. Ann Intern Med. 2015, 163:174-183. 10.7326/M14-2948

26. McKavanagh P, Lusk L, Ball PA, et al.: A comparison of cardiac computerized tomography and exercise stress electrocardiogram test for the investigation of stable chest pain: the clinical results of the CAPP randomized prospective trial. Eur Heart J Cardiovasc Imaging. 2015, 16:441-448. 10.1093/ehjci/jeu284

27. Dedic A, Lubbers MM, Schaap J, et al.: Coronary CT angiography for suspected ACS in the era of highsensitivity troponins: randomized multicenter study. J Am Coll Cardiol. 2016, 67:16-26. 10.1016/j.jacc.2015.10.045

28. Uretsky S, Argulian E, Supariwala A, et al.: Comparative effectiveness of coronary CT angiography vs stress cardiac imaging in patients following hospital admission for chest pain work-up: The Prospective First Evaluation in. Chest Pain (PERFECT) Trial. J Nucl Cardiol. 2017, 24:1267-1278. 10.1007/s12350-015-0354-6

29. Higgins JP, Altman DG, Gotzsche PC, et al.: The Cochrane Collaboration's tool for assessing risk of bias in randomised trials. BMJ. 2011, 343:5928. 10.1136/bmj.d5928

30. Newby DE, Adamson PD, Berry C, et al.: Coronary CT Angiography and 5-Year Risk of Myocardial Infarction. The. N Engl J Med. 2018, 379:924-933. 10.1056/NEJMoa1805971 